ATLAS Internal Note

INDET-NO-183

September 16, 1997

\title{
Radiation Hardness and Life Time Studies of LEDs and VCSELs for the Optical Readout of the ATLAS SCT
}

\author{
J. Beringer, K. Borer, R.K. Mommsen \\ Laboratory for High Energy Physics, University of Bern, Switzerland \\ E. Monnier \\ Centre de Physique des Particules de Marseille, CPPM \\ IN2P3 et Université d'Aix Marseille II, Marseille, France \\ R.B. Nickerson, A.R. Weidberg \\ Department of Nuclear Physics, Oxford University, UK \\ H.Q. Hou, K.L. Lear \\ Sandia National Laboratories, Albuquerque NM, USA
}

\begin{abstract}
We present the final results of radiation hardness and life time studies of Light Emitting Diodes (LEDs) and Vertical Cavity Surface Emitting Laser diodes (VCSELs). Following the encouraging results obtained in a previous life time test of neutron irradiated LEDs, we built an automatic measurement system in order to make long term tests with a large number of irradiated devices feasible. After irradiating about 170 LEDs from two different manufacturers and about 140 VCSELs with neutron and proton fluences as high (and in some cases about twice as high) as those expected at the inner tracker of ATLAS, we report on the radiation damage, the conditions required for its annealing, and we present post-irradiation failure rates for LEDs and VCSELs. The life time after irradiation was investigated by operating the diodes at an elevated temperature of $50^{\circ} \mathrm{C}$ for several months, resulting in operating times corresponding to up to 70 years of operation in the ATLAS SCT. From our measurements we calculate radiation damage constants and we find a protonto-neutron damage ratio of $3.2 \pm 0.1$ in GaAs for $24 \mathrm{GeV}$ protons and for the neutrons with a peak energy of about $1 \mathrm{MeV}$ available at the RAL ISIS facility. This result is in excellent agreement with recent NIEL calculations and provides an important check of the NIEL hypothesis.
\end{abstract}




\section{Contents}

1 Introduction $\quad 1$

2 Degradation of LEDs and VCSELs 2

2.1 Introduction . . . . . . . . . . . . . . . 2

2.2 Dark Line Defects . . . . . . . . . . . . . . . . 3

2.3 Temperature and Current Dependence of Life Time . . . . . . . . . . 3

2.4 Displacement Damage and Non-Ionizing Energy Loss . . . . . . . . . . 4

2.5 Radiation Damage in LEDs and VCSELs . . . . . . . . . . . . . . 6

2.6 Annealing of Radiation Damage . . . . . . . . . . . . . . 8

3 Devices $\quad 8$

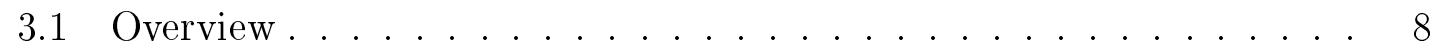

3.2 Light Emitting Diodes (LEDs) . . . . . . . . . . . . . . . 11

3.3 Vertical Cavity Surface Emitting Lasers (VCSELs) . . . . . . . . . 11

4 Experimental Procedure $\quad 16$

4.1 Overview . . . . . . . . . . . . . . . . . 16

4.2 The Scanning Machine . . . . . . . . . . . . . . . 16

4.3 Normalization and Calibration of the

Light Output Measurements . . . . . . . . . . . . . 20

4.4 Irradiation . . . . . . . . . . . . . . . . . . 20

4.5 Measurement Procedure for Irradiated Devices . . . . . . . . . . . 24

5 Results $\quad \mathbf{2 5}$

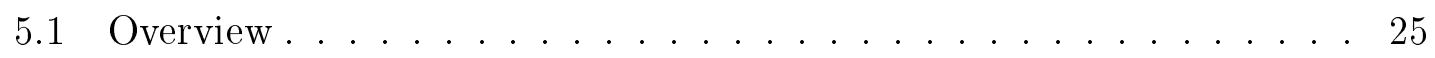

5.2 Damage Constants for GEC LEDs . . . . . . . . . . . . . . 25

5.3 Annealing of LEDs . . . . . . . . . . . . . . 26

5.4 Annealing of VCSELs . . . . . . . . . . . . . . . . . . 30

5.5 Reirradiation Studies . . . . . . . . . . . . . . . . . . . . 32

5.6 Lifetime Studies . . . . . . . . . . . . . . . . 36

5.7 Estimated Relative Light Output after Ten Years of Operation at LHC . . . . . . . . . . . . . 38

5.8 Statistics of Irradiated Devices . . . . . . . . . . . . . . . 38

5.9 Behavior of Failing Devices . . . . . . . . . . . . . . . 40

6 Conclusions 43

$\begin{array}{ll}\text { Acknowledgments } & 45\end{array}$

Bibliography $\quad 45$ 


\section{Introduction}

Two different technologies have recently been evaluated for the readout of the ATLAS SemiConductor Tracker (SCT): optical links and miniature shielded twisted pair cables (STP) as proposed for the readout of the Transition Radiation Tracker (TRT) [1]. In the case of optical links, one of the crucial issues is the radiation hardness and the life time of the optical emitters ${ }^{1}$, which would be mounted close to the SCT detector modules and thus be subject to fluences on the order of $10^{14}$ charged hadrons and $10^{14}$ neutrons per $\mathrm{cm}^{2}$, and to an ionizing dose of about $10^{5}$ Gy during 10 years of operation at LHC [1].

The radiation hardness and the long term reliability of different optical emitters suitable for a fibre optic link for the SCT, namely two types of Light Emitting Diodes (LEDs) from different manufacturers and one kind of Vertical Cavity Surface Emitting Laser diodes (VCSELs), have been studied in detail.

The scope of these studies has been twofold:

- The short term behavior of LEDs and VCSELs after irradiation was studied by irradiating them under different operating conditions with neutron and proton fluences comparable to (and in some cases exceeding) those expected at LHC. After the irradiation, the diodes were subjected to an annealing treatment which in most cases reduced the radiation damage considerably.

- The long term reliability after irradiation was studied in an accelerated life time test, where a large number of devices were operated at an elevated temperature of $50^{\circ} \mathrm{C}$ for several months. In order to reach the required statistics, a dedicated measurement system (the scanning machine) was built $[3,4]$, which allows for the long term operation and measurement of several hundred devices.

While it is well known that the light output of LEDs may be decreased after irradiation, little information seems to be available about the influence of radiation on the aging properties and thus on the life time of LEDs. For the LEDs typically used for fibre optic applications, i.e. high radiance devices operated at high current densities, the dominant degradation mechanism is the inhomogeneous development of crystal defects (dark line defects) acting as centers for non-radiative recombinations. Knowing that the development of dark line defects depends on initially present crystal defects and material impurities, the concern arises, that radiationinduced displacement damage in LEDs might lead to an increased growth of dark line defects and thus to a much shorter life time after irradiation.

Being a rather new type of device, the life time of VCSELs has been a major concern even without irradiation, and only recently VCSELs with a life time on the order of $10^{7}$ hours could be produced. As far as radiation hardness is concerned, little experimental data is available for VCSELs, although from theoretical considerations VCSELs can be expected to be very radiation hard devices.

\footnotetext{
${ }^{1}$ The radiation hardness of the PIN diodes used to receive the clock and command signals from the timing, trigger and clock distribution system (TTC) is of equal importance. Radiation hardness studies of PIN diodes are in progress at the University of Birmingham [2].
} 
In order to check if there is a large effect of irradiation on the life time of LEDs, we irradiated in an earlier life time test $[3,5]$ a small number of GaAlAs LEDs (manufactured by ABB Hafo) with up to $1.4 \cdot 10^{14} \mathrm{n} / \mathrm{cm}^{2}$. After the irradiation, an almost complete annealing of the radiation damage was observed under forward bias. Five LEDs were subsequently operated in an accelerated life time test without seeing any degradation due to aging for a duration which was estimated to correspond to about 57 years of operation in the ATLAS SCT.

Although these results were very encouraging, the conditions required for the annealing had to be clarified further, the tests had to be extended to include charged particle irradiation, and higher statistics were needed in order to make a prediction concerning the reliability of LEDs in the SCT. In addition, the use of VCSELs was suggested more recently as a cost effective and presumably more radiation hard replacement of the LEDs. Therefore we have continued our tests, studying both LEDs and VCSELs, and using an automatic measurement system in order to reach higher statistics. Complementary radiation hardness studies of LEDs have been performed by the University of Birmingham [6]. The radiation hardness of LEDs and of different types of laser diodes has also been studied by the ATLAS group developing the liquid argon calorimeter readout [7] and within the CMS collaboration [8].

While some first results were reported previously [9], we present in this paper the final results of our studies. The outline of this document is as follows: After a brief review of the mechanisms leading to the degradation of LEDs and VCSELs, we give some details about the devices studied in section 3 and we describe the experimental procedure and the scanning machine in section 4 . The results obtained are presented in detail in section 5 .

\section{Degradation of LEDs and VCSELs}

\section{$2.1 \quad$ Introduction}

In the following sections we attempt to give a brief review of the mechanisms leading to the degradation of LEDs and VCSELs as far as relevant for the present work, including both normal aging effects and the influence of irradiation. The aging processes leading to degradation and eventually to the failure of LEDs and VCSELs have been discussed in a large number of papers, application notes and textbooks (see e.g. [10]-[17] and references therein). In the following, only a short overview of the principal degradation mechanisms can be presented.

Typical operating life times of commercial unirradiated LEDs under normal operating conditions are on the order of $10^{5}-10^{7}$ hours, and it has been found experimentally that aging takes place only under forward bias conditions and not when the LED is turned off [10]. The data transmission protocol for the readout of the SCT was defined such that it would take advantage of this fact by switching the LEDs off when no data is transmitted [18]. 
For VCSEL, only a few life time studies of unirradiated devices are available. For the more common proton implanted VCSELs mean time to failure as high as $3 \cdot 10^{7}$ hours have been reported [19]. For oxide confined VCSELs the mean time to failure was estimated to be more than $3 \cdot 10^{5}$ hours $[20]$.

\subsection{Dark Line Defects}

For the LEDs typically used for fibre optic applications, i.e. high radiance devices operated at high current densities, the dominant degradation process is the inhomogeneous development of crystal defects acting as centers for non-radiative recombinations $[10,11,15,21]$. These defects, which occur also in semiconductor lasers, can be seen under high magnification as dark lines and are therefore often called Dark Line Defects (DLD).

The development of DLDs is due to the growth of dislocation networks by a climb mechanism under absorption or emission of point defects, apparently using the energy released under forward bias by non-radiative recombinations [22] - [28]. The growth and propagation of DLDs starts at initially present material impurities or crystal defects and, by increasing the non-radiative current, decreases the light output of the LED at a fixed forward current. The rate of growth increases with current density and temperature, but seems to be also enhanced by mechanical stress, e.g. due to diode assembly or dicing-induced strain [29, 30].

\subsection{Temperature and Current Dependence of Life Time}

The dependence of the mean life time ${ }^{2} \bar{t}$ of LEDs on operating temperature and current is usually given by an Arrhenius equation with a power law dependence on the forward current $[10,11,15]$

$$
\bar{t}=C I_{f}^{-n} \exp \frac{E_{a}}{k T_{j}}
$$

where $I_{f}$ is the forward current through the LED, $T_{j}$ denotes the junction temperature, and the constants $C, n$ and the thermal activation energy $E_{a}$ depend on the composition and the fabrication process of the device and must be determined experimentally. For LEDs, values found in the literature for $n$ and $E_{a}$ are typically in the range from 1 to 2 , and from $0.4 \mathrm{eV}$ to $1.0 \mathrm{eV}$, respectively.

The temperature dependence of $\bar{t}$ of VCSELs can be modelled by an Arrhenius equation, too, [31] and for proton implanted VCSELs, $E_{a}$ is typically in the range from $0.7 \mathrm{eV}$ to $1.1 \mathrm{eV}$ [16].

The current and temperature dependence of $\bar{t}$ may be used in a life time measurement in order to accelerate the aging and thus shorten the duration of the test. For some values of $E_{a}$, the acceleration factor that can be gained according to eq. (1) by raising the temperature with respect to aging at $T_{j}=10^{\circ} \mathrm{C}$ is shown in figure 1 .

\footnotetext{
${ }^{2}$ According to current industry practice, a LED is considered as dead when the light output has dropped below $50 \%$ of the initial light output.
} 


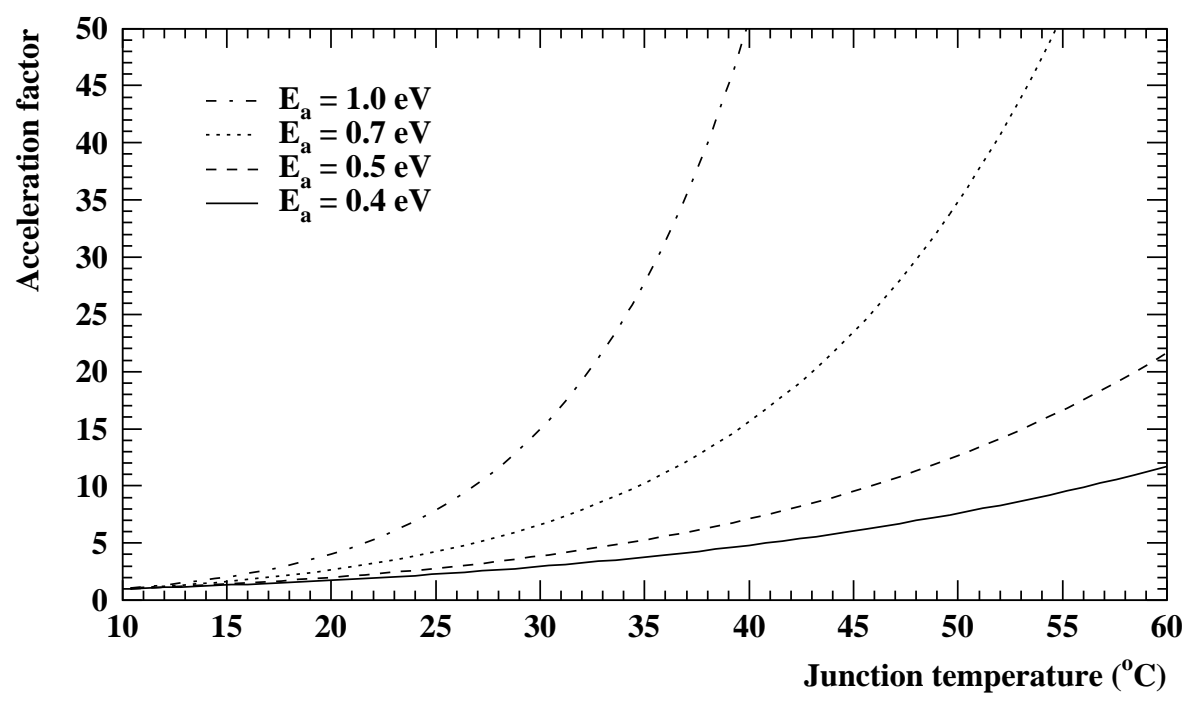

Figure 1: Acceleration factors for different thermal activation energies $E_{a}$, calculated with respect to aging at $T_{j}=10^{\circ} \mathrm{C}$.

\subsection{Displacement Damage and Non-Ionizing Energy Loss}

The degradation of LEDs and VCSELs by irradiation is primarily due to displacement damage (bulk damage). If a high energy particle interacts with an atom (called the primary knock-on atom, PKA) in the semiconductor lattice, enough kinetic energy may be transferred to dislodge the atom from its site. The PKA will rapidly loose its energy in the vicinity of the primary interaction site due to both ionization and the displacement of further atoms, eventually producing a cascade of collision processes. Energetic charged particles or fragments produced in inelastic nuclear collisions may contribute to the resulting displacement damage, too, and very low energy neutrons may cause displacement damage through nuclear reactions like e.g. $(n, \gamma)$ even if their kinetic energy is below the elastic threshold of about $200 \mathrm{eV}$ in GaAs. Even though in the end only a small fraction ${ }^{3}$ of the total energy deposition goes into the displacement of atoms (non-ionizing energy loss, NIEL), depending on the energy of the PKA, a large number of crystal defects may be produced in a tree-like structure $[34,35,36]$.

The initial displacement damage is produced on the fast collision time scale of about $10^{-14} \mathrm{~s}$ to $10^{-12} \mathrm{~s}[35]$ and is complete before any thermally activated atomic motions take place. At a slower time scale, the generated vacancies and interstitials will move around and most of them $(\sim 95 \%)$ will recombine due to diffusion processes (short term annealing). Those that remain will eventually form a variety of stable defect complexes, including di-vacancies, vacancy-impurity complexes, and larger clusters. Depending on the temperature, further annealing [37], but also reverse annealing may take place. The annealing processes may be enhanced under forward

\footnotetext{
${ }^{3}$ The distribution of the energy deposition between ionizing and non-ionizing processes is described by the Lindhard theory [32, 33].
} 
bias (injection annealing) [22], because electron-hole recombinations at a defect can increase the defect's mobility.

The presence of defect complexes changes the effective doping concentration and, by introducing additional states within the forbidden bandgap of the semiconductor, they act as recombination centers, possibly causing a decrease of the carrier density, the minority carrier life time, and the carrier mobility.

The different defect complexes might be expected to have different probabilities for their formation, depending on the type and energy of the radiation, and to possess different efficiencies for producing macroscopic device degradation. However, it has been observed that the degradation due to displacement damage of a given semiconductor device at specific operating conditions is primarily a function of the non-ionizing energy deposition (NIEL hypothesis) and not of the particular type or energy of the incident particles. Although some deviations of measured device degradation from the one expected according to the NIEL hypothesis have been reported (see e.g. [38, 39, 40]), the NIEL hypothesis has been verified experimentally over a wide range of energies, for different incident particles, for silicon and to a somewhat less degree for GaAs [38] - [43].

For a particle passing through a given material, the NIEL $^{4}$ is given by

$$
\frac{d E}{d x}_{N}=\frac{N_{a}}{A^{\prime}} \sum_{Z, A} \int E_{r} \frac{d \sigma}{d E_{r}} L\left(E_{r}\right) d E_{r}
$$

where $d \sigma / d E_{r}$ is the differential cross section for producing a recoil atom or fragment with energy $E_{r}$, atomic weight $A$ and atomic number $Z$, and the sum extends over all types of recoil atoms and fragments. $L\left(E_{r}\right)$ is the Lindhard partition function [32], which gives the fraction of the recoil energy $E_{r}$ that contributes to displacement damage. $N_{a}$ and $A^{\prime}$ are Avogadro's number and the atomic weight of the material, respectively. NIEL calculations are available for different particles both for Si [41, 44, 45, 46] and for GaAs [41, 45, 47], although for charged pions, which dominate the radiation environment in the SCT near the interaction point, and, to a smaller extent for protons, these calculations presently have rather large uncertainties [48]. Table 1 summarizes some values of the NIEL in GaAs.

By comparing the NIEL with the one of particles chosen as a reference - like e.g. $1 \mathrm{MeV}$ neutrons or $24 \mathrm{GeV}$ protons - damage functions can be defined which correlate the displacement damage produced by different particles at different energies in a given semiconductor material. Such damage functions have been calculated by several authors for different incident particles and semiconductor materials [44, 45, 46]. Using these calculations, one can e.g. calculate for a given radiation environment an equivalent $1 \mathrm{MeV}$ neutron fluence, which is expected to produce the same amount of displacement damage, or the results of radiation hardness tests can be scaled for a particular application. For example, the NIEL values for $24 \mathrm{GeV}$ protons and $300 \mathrm{MeV}$ pions are equivalent within the large uncertainties. Therefore, $24 \mathrm{GeV}$ proton fluences can be considered as good approximation to the dominating $300 \mathrm{MeV}$

\footnotetext{
${ }^{4}$ Except for the factor $N_{a} / A^{\prime}$, the NIEL is equivalent to the KERMA (kinetic energy released in the material) cross sections.
} 


\begin{tabular}{|l|c|c|c|}
\hline $\begin{array}{l}\text { Particle } \\
\text { and energy }\end{array}$ & $\begin{array}{c}\text { NIEL } \\
\left(\mathrm{keV} \mathrm{cm}^{2} / \mathrm{g}\right)\end{array}$ & $\begin{array}{c}\text { Ratio to } \\
1 \mathrm{MeV} \mathrm{n}\end{array}$ & $\begin{array}{c}\text { Ratio to } \\
24 \mathrm{GeV} \mathrm{p}\end{array}$ \\
\hline \hline 1 MeV neutrons & 0.55 & 1.0 & 0.2 \\
\hline ISIS neutrons & 0.9 & 1.6 & 0.3 \\
\hline Dynamitron neutrons & 0.68 & 1.2 & 0.2 \\
\hline $24 \mathrm{GeV}$ protons & 2.9 & 5.3 & 1.0 \\
\hline $300 \mathrm{MeV}$ pions & 3.6 & 6.5 & 1.2 \\
\hline
\end{tabular}

Table 1: NIEL in GaAs neutrons, protons and pions at different energies (from [47, 49] and references quoted therein). The NIEL quoted for ISIS neutrons and Dynamitron neutrons takes the energy spectrum of the ISIS and Dynamitron neutron irradiation facilities into account (see section 4.4). The uncertainty of the NIEL given for $1 \mathrm{MeV}$ neutrons is $\sim 10 \%$, while for $24 \mathrm{GeV}$ protons and pions an uncertainty on the order of a factor of 2 is not unlikely.

pions in the ATLAS SCT environment. However, it must be emphasized that by doing so one relies on the validity of the NIEL hypothesis which should therefore be checked thoroughly. Further it should be noted that effects from non-local energy deposition outside a device's sensitive volume may be essential for high energy protons, and any additional long term ionization effects must be taken into account in order to obtain good agreement of the damage functions with the experimental data.

While the displacement damage produced by different particles at different energies relative to e.g. $1 \mathrm{MeV}$ neutrons is primarily a function of the NIEL, the degradation of a particular semiconductor device by a given amount of irradiation, measured as a change in a specific performance parameter, depends, of course, on many additional factors. These include e.g. the sensitivity of the device to a reduction of minority carrier life time, carrier density and mobility, the doping and impurity concentrations, and the influence on the annealing processes of temperature, radiation flux and biasing conditions during and after the irradiation.

\subsection{Radiation Damage in LEDs and VCSELs}

The most important effect of radiation on LEDs is the introduction of stable defect complexes which act as non-radiative recombination centers (see e.g. [50, 51]), so that, at a fixed operating current, the fraction of non-radiative recombinations is increased and therefore the minority carrier life time $\tau$ decreases. Thus, the minority carrier life time is sensitive to the non-ionizing energy loss (NIEL) inside the active semiconductor area and can be used to verify experimentally the NIEL hypothesis.

The total pre-irradiation minority carrier life time $\tau_{0}$ may be written as

$$
\frac{1}{\tau_{0}}=\frac{1}{\tau_{r}}+\frac{1}{\tau_{n r}}
$$


where $\tau_{r}$ and $\tau_{n r}$ denote the initial life times associated with radiative and nonradiative processes, respectively. The radiation-induced non-radiative recombination centers decrease the minority carrier life time $\tau$,

$$
\frac{1}{\tau}=\frac{1}{\tau_{0}}+\sum_{i} v_{t h} \sigma_{i} n_{i}
$$

where $v_{t h}$ is the average thermal velocity of the minority carriers, $n_{i}$ and $\sigma_{i}$ denote the density and capture cross section, respectively, of the defect complexes of a given type, and the sum extends over the different types of radiation-induced defects. As long as there is no significant overlap of the regions where the individual incident particles produce crystal defects ${ }^{5}$, the resulting initial ${ }^{6}$ defect densities $n_{i}$ will be proportional to the fluence $\phi$ (or, equivalently, to the dose),

$$
n_{i}=c_{i} \phi
$$

where the coefficients $c_{i}$ denote the density of defects of a given type produced per unit fluence.

Defining a damage constant $K$,

$$
K=v_{t h} \sum_{i} c_{i} \sigma_{i}
$$

eq. (4) can be rewritten as

$$
\frac{\tau_{0}}{\tau}=1+\tau_{0} K \phi
$$

If it is assumed that the total current density in the LED junction is dominated by diffusion currents, the ratio $\tau_{0} / \tau$ can be directly related to the relative light output $R L O$ after irradiation [50]:

$$
\left(\frac{1}{R L O}\right)^{\frac{2}{3}}=\frac{\tau_{0}}{\tau}=1+\tau_{0} K \phi
$$

This relation shows that a degradation of the minority carrier life time $\tau$, and thus of the light output, is expected if the product $\tau_{0} K \phi$ becomes significant compared to 1 . The damage constant $K$ is determined by the NIEL of the radiation, by the composition of the semiconductor material, and by the amount of annealing that has taken place. Therefore $K$ cannot be changed easily in order to obtain more radiation hard LEDs. However, the pre-irradiation minority carrier life time $\tau_{0}$ may be minimized by increasing the radiative recombinations through heavily doping the optical emitting region and by operating the LED at high current densities [51]. This has not only the advantage of increasing the radiation hardness, but improves also the light yield and the speed of the LED.

\footnotetext{
${ }^{5}$ For neutron irradiated silicon, it has been estimated that nonlinear effects should not occur below fluences of $\sim 2.5 \cdot 10^{15} \mathrm{n} / \mathrm{cm}^{2}[52]$.

${ }^{6}$ If annealing takes place, the densities $n_{i}$ will change as defects disappear or are transformed into different defect complexes, possibly leading to nonlinear effects in $\phi$ if more than one defect participates in a defect reaction.
} 
Since for VCSELs the minority carrier life time in the lasing regime is dominated by stimulated emission, it is several orders of magnitude smaller than for LEDs and much higher fluences are required to produce a substantial change of $\tau_{0} / \tau$. Below the lasing threshold, however, the minority carrier life time is determined by spontaneous emission as is the case for LEDs. Therefore the presence of radiation induced nonradiative recombination centers decreases the light yield and higher currents are required to enter the lasing regime. Thus the lasing threshold current of VCSELs is expected to increase after irradiation.

\subsection{Annealing of Radiation Damage}

Significant annealing of radiation damage of LEDs under forward bias (injection annealing) has been reported for different types of radiation and LEDs (and also for laser diodes) by several authors $[5,50,53,54,55,56]$, but in some special cases, mostly concerning $\mathrm{SiC}$ and $\mathrm{Si}$-doped GaAs devices, no annealing could be obtained $[50,57]$. In GaAs $p^{+} n$ junctions defect annealing under forward bias was studied after $1 \mathrm{MeV}$ electron irradiation [22] with Deep Level Transient Spectroscopy [58], and it was found that the annealing process is directly related to recombinations at the defect.

Thermal annealing of LEDs was reported, too, but seems to require temperatures above $200^{\circ} \mathrm{C}$ for the annealing of defects which are stable at room temperature $[37,55]$.

\section{Devices}

\subsection{Overview}

In our tests we studied two types of Light Emitting Diodes (LEDs) from ABB Hafo (Sweden) and from GEC-Marconi (U.K.), respectively, and Vertical Cavity Surface Emitting Laser Diodes (VCSELs) from Sandia National Laboratories, Albuquerque (NM, USA). An overview of the devices is given in table 2. Except for the different packaging, the ABB LEDs correspond to those used in the previous life time test $[3,5]$, where they showed an excellent radiation hardness under neutron irradiation. The GEC LEDs are custom devices, which were developed together with a dedicated radiation hard package for possible use at LHC. The kind of VCSELs tested is still under development and we examined devices from three different production lots.

All devices were purchased without optical fibres and without any packaging, and were mounted on ceramic boards serving as carriers for the operation in the scanning machine. A picture of the different types of devices mounted on the ceramic boards used in the scanning machine are shown in figure 9 (section 4, p. 18).

Before the irradiation, the devices were installed in the scanning machine (see section 4.2) and an initial characterization of the devices was performed. The default measurement conditions and the electrical characteristics of the devices are summarized in table 3 and will be described in the following sections in more detail. 


\begin{tabular}{|c|c|c|c|}
\hline Label & Description & Manufacturer & Comments \\
\hline \hline ABB LEDs & GaAlAs LEDs & ABB Hafo (Sweden) & $\begin{array}{c}\text { single device, } \\
\text { no longer produced }\end{array}$ \\
\hline GEC LEDs & GaAlAs LEDs & GEC-Marconi (U.K.) & $\begin{array}{c}\text { single device, } \\
\text { custom manufactured }\end{array}$ \\
\hline VCSELs Ia & $\begin{array}{c}\text { GaAs } / \text { GaAlAs } \\
\text { VCSELs } \\
I_{t h} \lesssim 2 \mathrm{~mA}\end{array}$ & $\begin{array}{c}\text { Sandia National } \\
\text { Laboratories (USA) }\end{array}$ & $\begin{array}{c}\text { twenty-fold arrays, } \\
\text { in development }\end{array}$ \\
\hline VCSELs Ib & $\begin{array}{c}\text { GaAs } / \mathrm{GaAlAs} \\
\text { VCSELs } \\
I_{t h} \simeq 2 \mathrm{~mA}\end{array}$ & $\begin{array}{c}\text { Sandia National } \\
\text { Laboratories (USA) }\end{array}$ & $\begin{array}{c}\text { twenty-fold arrays, } \\
\text { in development }\end{array}$ \\
\hline VCSELs II & $\begin{array}{c}\text { GaAs } / \mathrm{GaAlAs} \\
\text { VCSELs } \\
I_{t h} \lesssim 1 \mathrm{~mA}\end{array}$ & $\begin{array}{c}\text { Sandia National } \\
\text { Laboratories (USA) }\end{array}$ & $\begin{array}{c}\text { twenty-fold arrays, } \\
\text { in development }\end{array}$ \\
\hline
\end{tabular}

Table 2: Overview of devices used for the irradiation studies presented in this work
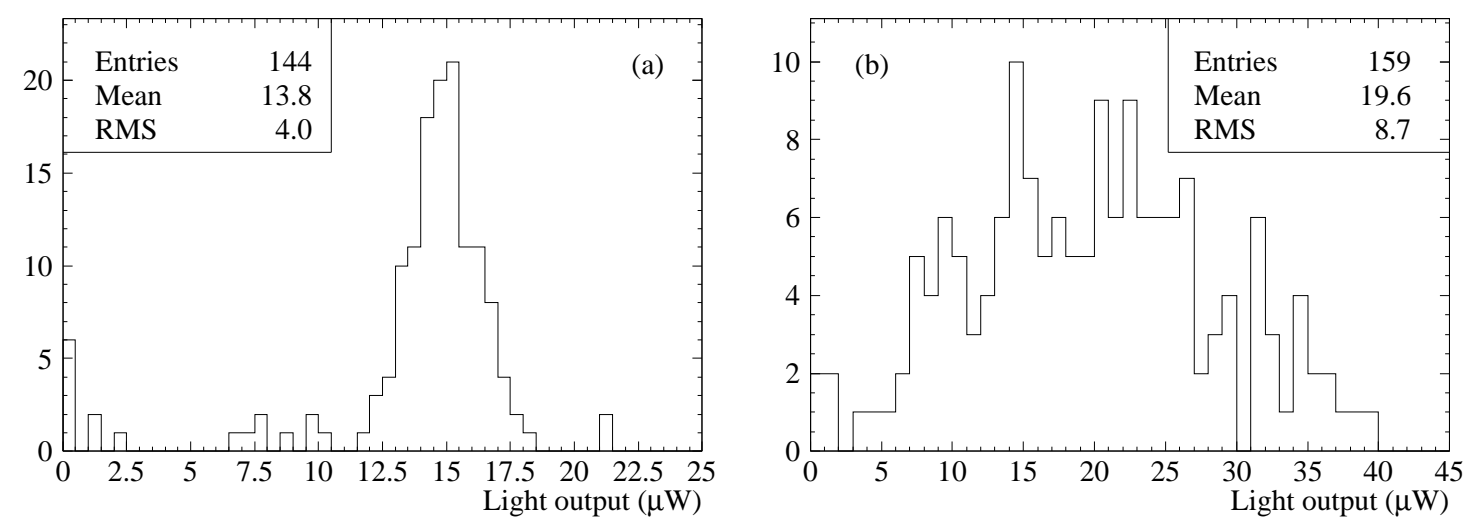

Figure 2: Distribution of the light output at $+10^{\circ} \mathrm{C}$ (a) of the ABB LEDs at $10 \mathrm{~mA}$ forward current and (b) of the GEC LEDs at $20 \mathrm{~mA}$ forward current. 


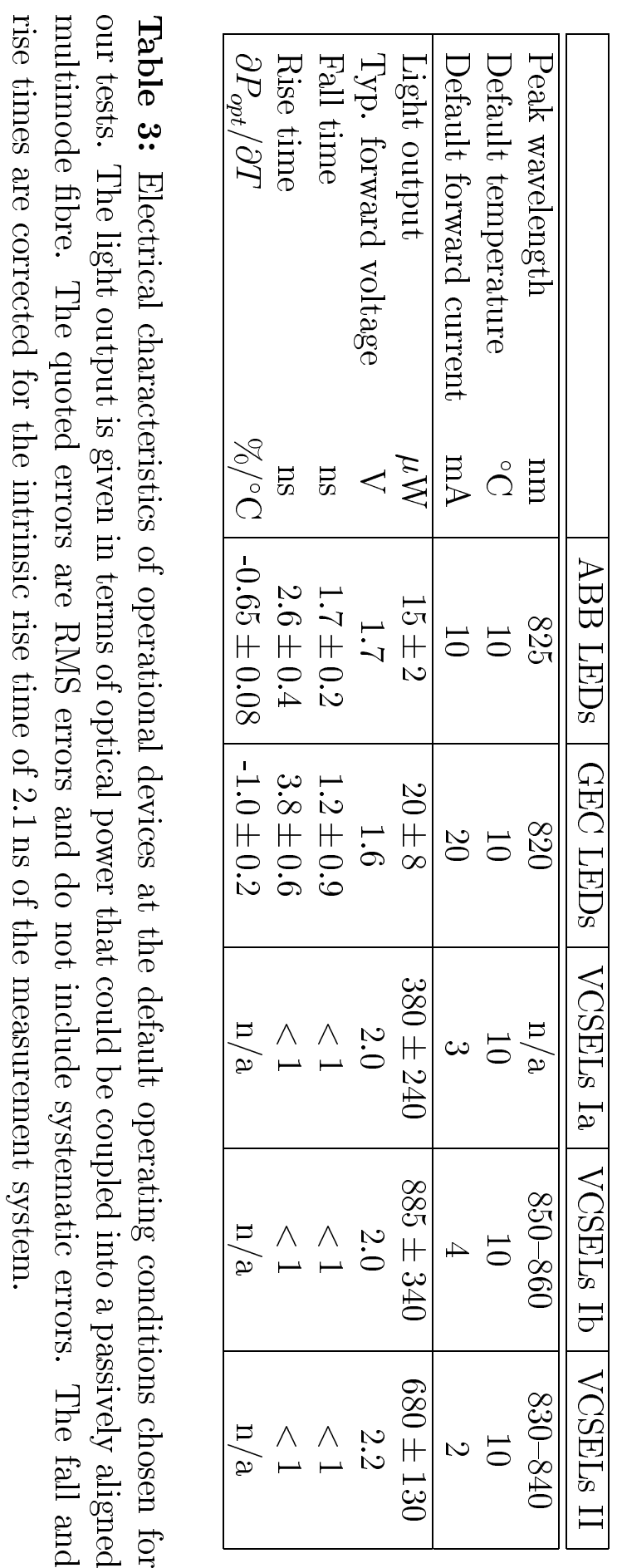




\subsection{Light Emitting Diodes}

\subsubsection{Required Light Output}

The LEDs have to provide an initial optical power of at least $10 \mu \mathrm{W}$ into a multimode fibre in order to make sure that the optical links remain operational during 10 years at LHC in spite of the anticipated degradation [1]. Since not all of the LEDs purchased were tested by the manufacturer, not all LEDs satisfied this requirement at the default operating current as can be seen from figure 2. For this test only devices with a light output of less than $5 \mu \mathrm{W}$ were rejected for not loosing too much in statistics.

\subsubsection{ABB Hafo LEDs}

The ABB LEDs were chosen initially for possible use in the ATLAS SCT because of their radiation hardness, their outstanding light yield, and their fast response. However, they are not available commercially in a package which is suitable for the application in the SCT. Recently, ABB Hafo was sold to Mitel Corporation and the production of this LED type was stopped.

The distribution of the pre-irradiation light output of all 144 ABB LEDs at $10 \mathrm{~mA}$ and $+10^{\circ} \mathrm{C}$ is shown in figure $2 \mathrm{a}$. Due to the fact, that the ABB LEDs were tested by ABB Hafo, all devices were working. However, 9 out of 144 LEDs yielded less than $5 \mu \mathrm{W}$ of optical power and were screened out.

\subsubsection{GEC-Marconi LEDs}

The second type of LED tested was developed by GEC-Marconi (U.K.) on behalf of the Oxford group. These LEDs were specially designed for use in the SCT, and a suitable radiation hard package is available. However, in contrast to the ABB LEDs, they show a marked threshold effect and produce little light below a forward current of about $10 \mathrm{~mA}$. Therefore, a $20 \mathrm{~mA}$ forward current was chosen as the default operation current (see table 3 ).

The GEC LEDs were not tested by the manufacturer, and 17 out of the 176 tested devices gave no light at all. This is mainly due to mechanical defects like broken or not existing bonding wires. The distribution of the light output is shown in figure $2 \mathrm{~b}$. As can be seen another six devices were below the $5 \mu \mathrm{W}$ threshold and had to be screened out.

\subsection{Vertical Cavity Surface Emitting Lasers}

\subsubsection{VCSELs from Sandia National Laboratories}

Vertical Cavity Surface Emitting Lasers (VCSELs) are an attractive alternative to LEDs as emitters for an optical link. In contrast to the older edge emitting lasers, the mirror is grown as paired layers of semiconductor materials into the laser structure itself. Therefore, no mirrors have to be cleaved out of the crystalline structure, making the production and the testing of laser diodes much easier, since testing is possible already on the wafer. 


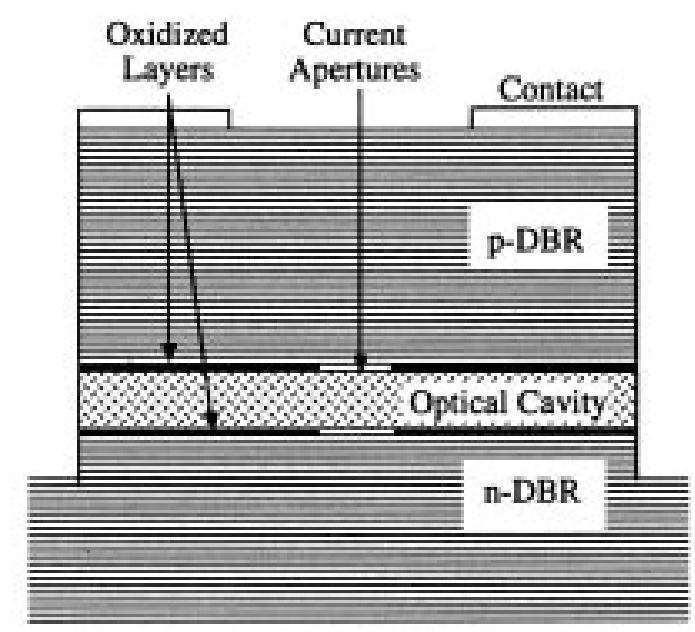

Figure 3: Sketch of an oxide-confined VCSEL with distributed Bragg reflectors (DBR) grown above and below the optical cavity. The lateral oxidized layers confine the current to a small region of the cavity.

Three types of VCSELs from three different production runs were purchased from Sandia National Laboratories, Albuquerque (NM, USA), in collaboration with the ATLAS group developing the liquid argon calorimeter readout [59]. The three types are labeled by roman numbers in the following. At $+10^{\circ} \mathrm{C}$ the lasing threshold current is about $\sim 2 \mathrm{~mA}$ (or a bit less) for VCSEL types Ia and Ib, and a few devices have a threshold current below $1 \mathrm{~mA}$ (VCSEL type II). Due to the different lasing threshold currents, each type of VCSEL was assigned its own default forward current as listed in table 3. As can be seen in the table, the spread of the light output of the individual devices is large for all three types of VCSELs. The fall and rise time within the lasing regime is much faster than the one of the electronics of the scanning machine $(\sim 2 \mathrm{~ns})$. The thermal behavior of VCSELs depends strongly on the design of the device and will be discussed in section 3.3.2.

In contrast to the more commonly available proton implanted VCSELs, the VCSELs used in our test were made with a selective oxidation technique [60]. Figure 3 shows a sketch of an oxide confined VCSEL. The oxidized layers confine the current to a small region of the optical cavity, increasing the current density and thus decreasing the lasing threshold. Above and below the optical cavity, distributed Bragg reflector (DBR) mirrors are built from interleaved layers of GaAs and $\mathrm{Al}_{\mathrm{x}} \mathrm{Ga}_{1-\mathrm{x}}$ As. The variable $\mathrm{x}$ parameterizes the refractive index and ranges from 0.96 in the innermost to 0.16 in the outermost layers.

All VCSELs tested were manufactured in the form of twentyfold arrays and mounted onto ceramic boards for use in the scanning machine. However, the geometrical arrangement of the driver electronics in the scanning machine is not adapted to these arrays and only 8 electronics channels are available for each ceramic board. 
Thus only 8 out to the 20 VCSELs can be operated simultaneously, although by shifting the ceramic board relative to its connector another 8 devices become accessible. Therefore, 16 out of the 20 devices on each array could be studied.

\subsubsection{Temperature Dependence of the Lasing Threshold Current}

The lasing threshold current of VCSELs is determined by the optical gain at the lasing wavelength. Since both the Fabry-Perot cavity wavelength and the gain spectrum depend on the operating temperature, the lasing threshold current is not necessarily a monotonic function of temperature ${ }^{7}$, in contrast to most edge-emitting lasers. However, for each type of VCSEL there is an optimal operating temperature where an ideal alignment between the gain spectrum and the lasing wavelength takes place, which, in most cases results in a minimum threshold current. Therefore, VCSELs may be tuned for a given operating temperature.

The VCSELs used in our test were optimized for room temperature operation, and they show a very uneven behavior at $-10^{\circ} \mathrm{C}$. However, similar VCSELs could be produced for operation at $-10^{\circ} \mathrm{C}$, and recent developments lead to laterally oxidized VCSELs with a sub-milliamp threshold current and good light output in a temperature range from $77 \mathrm{~K}$ to $370 \mathrm{~K}$ [62].

\subsubsection{Modal Noise and Transverse Modes}

VCSEL cavities are tuned to lase in a single longitudinal mode. However, in the presence of reflective external surfaces like e.g. an uncoated optical fibre, the effective length of the laser cavity may be altered, possibly giving rise to additional longitudinal modes. This effect was observed for feedback strengths as low as $1 \%$ [63], and the modal noise induced by reflections was increased in some cases by more than $40 \mathrm{~dB}$ above the noise level observed without any feedback [64].

When measuring VCSELs in the scanning machine, a large amount of noise was observed initially, presumably due to backreflection from the lens built into the case of the PIN diode used for measuring the light output. A filter with an attenuation of a factor 8 at a wavelength of about $850 \mathrm{~nm}$ placed on top of the PIN diode improved the signal quality considerably, as can be seen in figure 4. Therefore, all VCSEL measurements were subsequently made with filters with attenuation factors between about 8 and 22 .

Another well known problem of VCSELs concerns the development of higher order transverse modes. Due to the lateral diameter of the active optical cavity of typically $>5 \mu \mathrm{m}$, which is large compared to the lasing wavelength of $\sim 0.3 \mu \mathrm{m}$ in GaAs, higher order transverse electromagnetic modes (TEM) may develop. Since the reflectivity of the distributed Bragg mirrors becomes lower for higher order TEM modes, the ground mode is strongly favored [65]. Nevertheless, at elevated forward currents (i.e. for higher pump levels), higher order TEM modes may appear due to different effects like e.g. spatial hole burning of the lateral gain profile due to thermal lensing [66]-[70].

\footnotetext{
${ }^{7} \mathrm{~A}$ comprehensive review of thermal effects in VCSELs may be found in [61].
} 


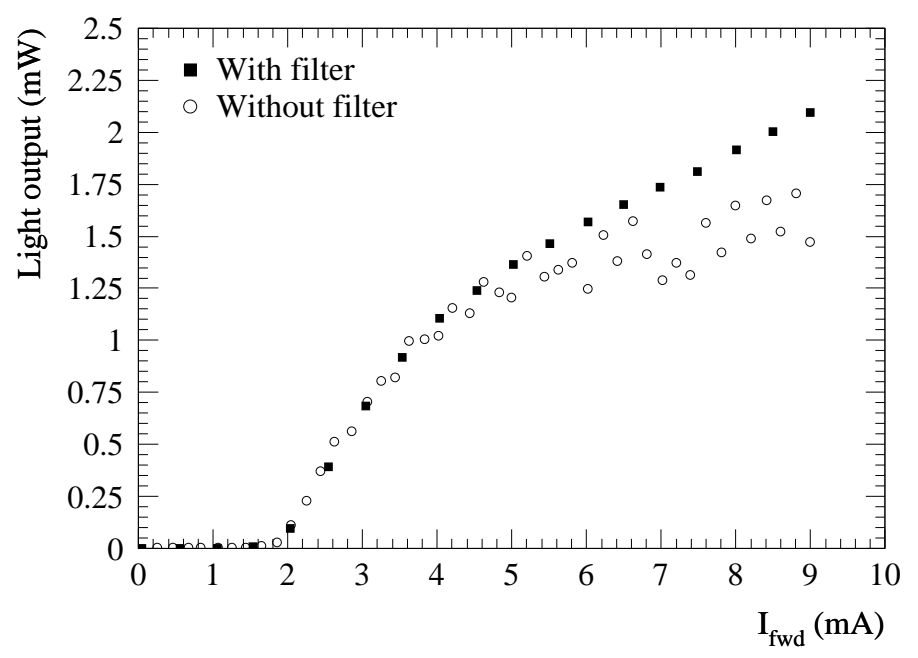

Figure 4: L-I curve with and without modal noise from feedback (see text)

Figure 5 shows the transverse light output profile of a single VCSEL of type $\mathrm{Ib}$ as a function of the forward current as measured in the scanning machine with the PIN diode placed at a distance of a few millimeters in front of the VCSEL. The development of higher order transverse modes above $2 \mathrm{~mA}$ is clearly visible. In the corresponding light output vs. current curve (L-I curve), the development of higher order modes manifests itself as characteristic changes in the slope whenever a new transverse mode starts to develop (figure 6a). If not all of the emitted light is collected, e.g. because the PIN diode is placed too far away from the VCSEL or because of a misaligned optical fibre, the development of higher order transverse modes may lead to a non-monotonic dependence of the light output from the forward current as shown in figure $6 \mathrm{~b}$.

Both the modal noise and the development of higher order transverse modes represent a potential problem for the coupling of VCSELs to fibres. One solution is to use a special optical interface like e.g. the GUIDECAST used in Motorola's OPTOBUS system [71]. The drawback of such a solution is the increased cost and the possible lack of radiation hardness of the interface. However, a passively self-aligned plastic package with a MT connector for a 16 channel two-dimensional VCSEL array has recently been developed $[72,73]$ and a bit error rate of $10^{-11}$ was achieved for a $1 \mathrm{~Gb} / \mathrm{s}$ optical link. The development of radiation hard VCSEL-tofibre connectors suitable for the application in ATLAS is currently pursued by the group developing an optical readout for the liquid argon calorimeter [74]. 

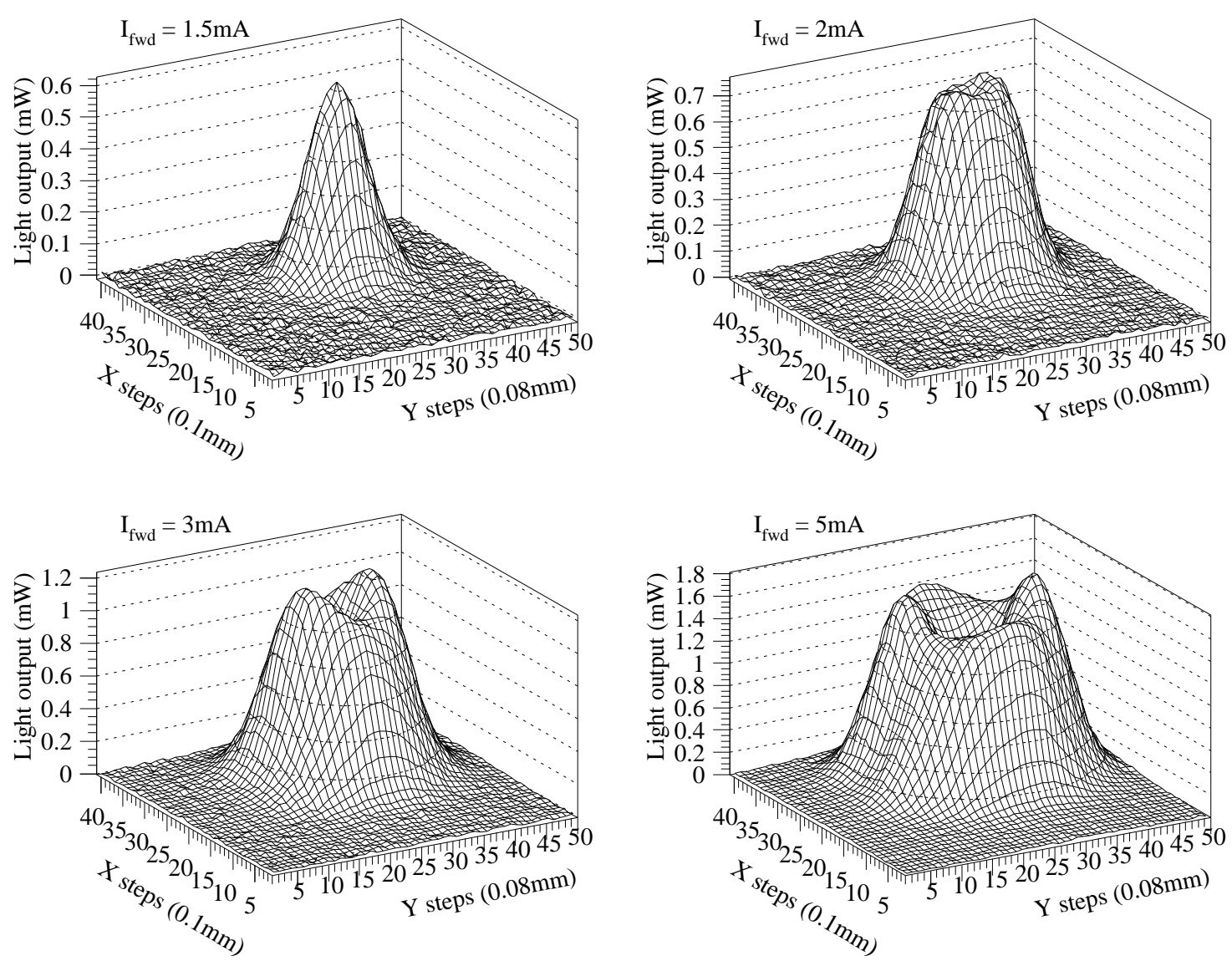

Figure 5: Transverse light output profile of a VCSEL of type Ib as a function of the forward current. Above $\sim 2 \mathrm{~mA}$ higher order transverse modes develop. The corresponding L-I curve is shown below in figure $6 \mathrm{a}$.
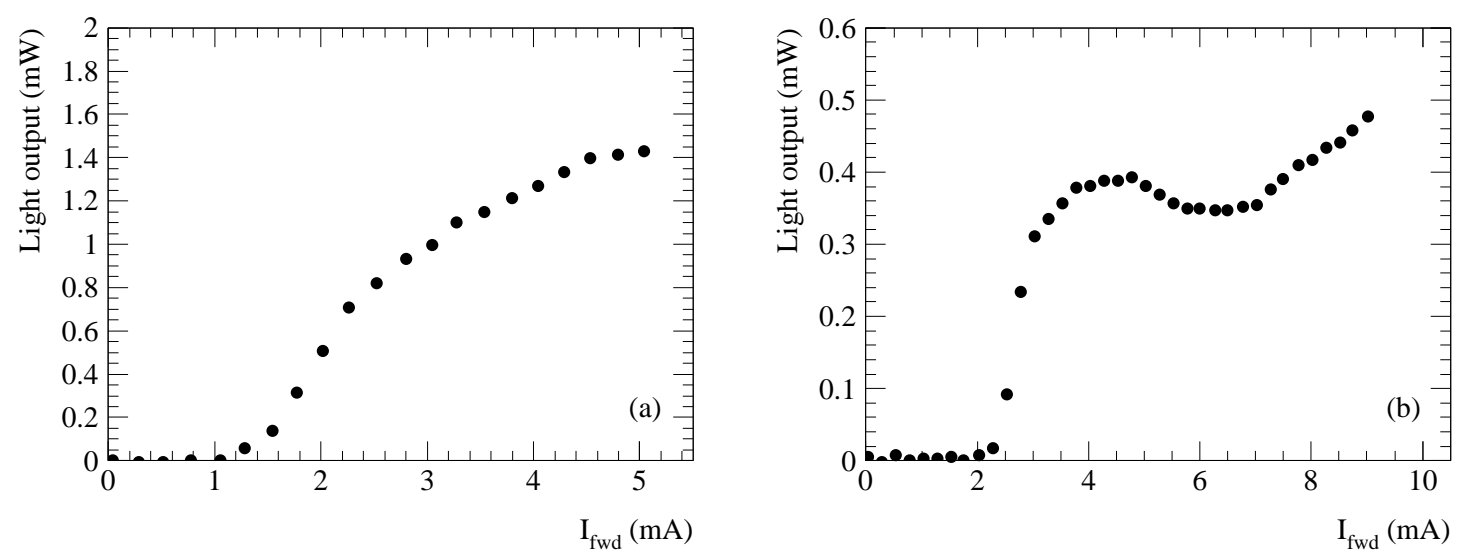

Figure 6: a) L-I curve for the VCSEL of type Ib whose transverse light output profile is shown above. b) L-I curve for a different VCSEL of type Ia where higher order transverse modes start to develop at about $4 \mathrm{~mA}$ to $5 \mathrm{~mA}$. The strange non-monotonic behavior arises because the PIN diode is not close enough to the VCSEL to collect all of the light emitted. 


\section{Experimental Procedure}

\subsection{Overview}

In order to make the necessary long term measurements of a large number of irradiated LEDs feasible, an automatic measurement system, called the scanning machine, was built with space and driver circuits for 448 LEDs or VCSELs.

Before the irradiation, the devices were temporarily installed in the scanning machine for measuring the light output as a function of the forward current and the operating temperature. Most of the ABB LEDs were subjected to a burn-in treatment at $50 \mathrm{~mA}$ and $50^{\circ} \mathrm{C}$ before the irradiation. Unfortunately, due to time constraints, no burn-in period could be allocated for the GEC LEDs and for the VCSELs. After the irradiation, the devices were reinstalled in the scanning machine. Following a first measurement at the default conditions (see table 3 ), the devices were operated at different forward currents and at either $+10^{\circ} \mathrm{C}$ or $-10^{\circ} \mathrm{C}$ for several days or weeks in order to investigate the annealing behavior. During this annealing period, frequent measurements were made. In order to investigate the long-term reliability of the irradiated devices, they were operated (and measured regularly) for several months at an elevated temperature of $50^{\circ} \mathrm{C}$ in order to accelerate the aging.

\subsection{The Scanning Machine}

A picture of the scanning machine with its electronics and with the data acquisition system is shown in figure 7 .

Since in the first test considerable inaccuracy and handling problems were introduced by the use of optical fibres and connectors, and since the radiation hardness of optical fibres was sufficiently demonstrated [56], the light output of the individual LEDs and VCSELs is measured in the scanning machine directly with a moving PIN photodiode at a distance of a few millimeters in front of the diodes, thus avoiding the need of optical fibres.

A longitudinal sectional view of the scanning machine is shown in figure 8. Two shifters are used to move an optical receiver board with two PIN photodiodes (one for measuring the LEDs and the other equipped with a filter for the much brighter VCSELs) in the longitudinal (X) and transverse (Y) directions in steps of $0.1 \mathrm{~mm}$ and $0.04 \mathrm{~mm}$, respectively. The LEDs or VCSELs are mounted on temperature controlled supports called blocks (figure 10), emitting the light downwards to the PIN diodes. A precise mechanical mounting ensures a well defined vertical distance (which can be adjusted for each block as needed) between the devices and the PIN diodes. By moving the PIN diode to the position yielding the maximum signal below a given device, the measurement of the light output of the diodes is reproducible to about $2 \%$.

The scanning machine is equipped with 7 blocks for which the temperature can be set individually between $-10^{\circ} \mathrm{C}$ and $50^{\circ} \mathrm{C}$ using Peltier elements for cooling or heating. In order to keep the humidity low and to protect the diodes from ice at low temperatures, the scanning machine provides a tight volume with a nitrogen atmosphere. 


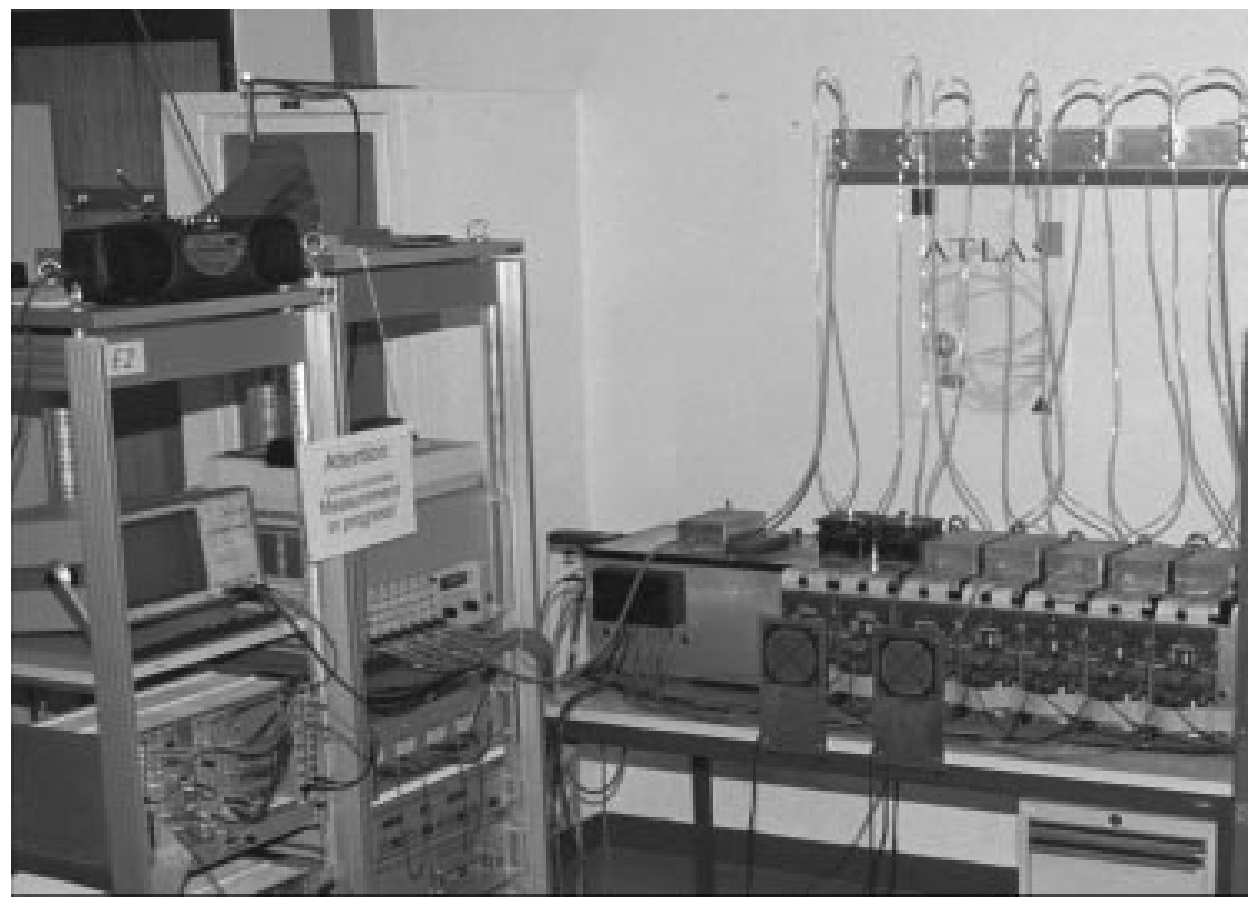

Figure 7: The Scanning Machine installed in the laboratory (right) and its associated electronics (left)

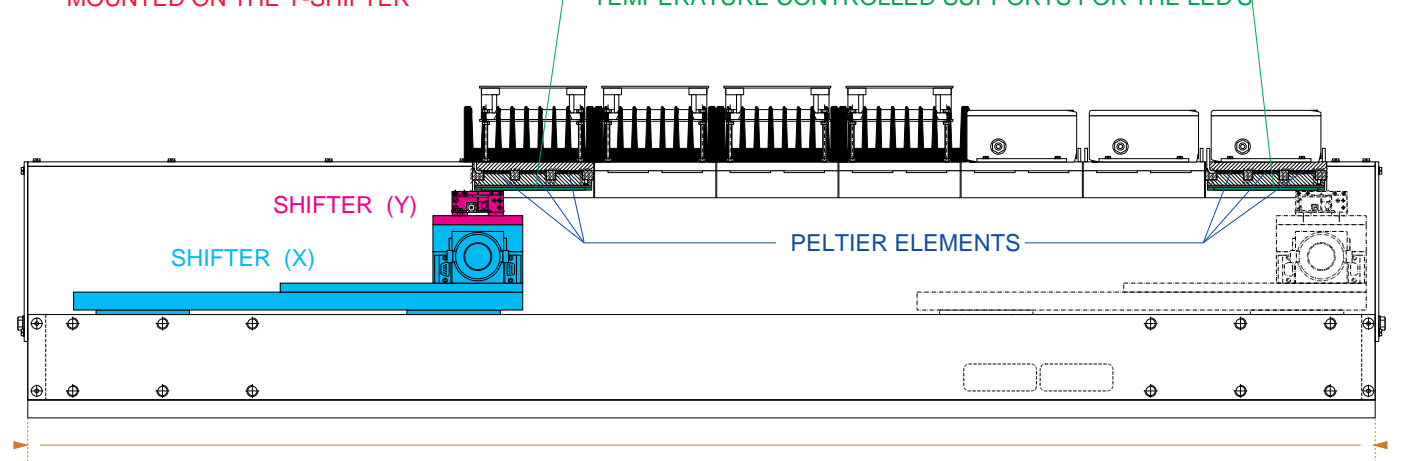

$1505 \mathrm{~mm}$

Figure 8: Longitudinal sectional view of the scanning machine. The shifter moving in the longitudinal $(\mathrm{X})$ direction is shown both at the leftmost (shaded) and at the rightmost (dashed lines) position. 

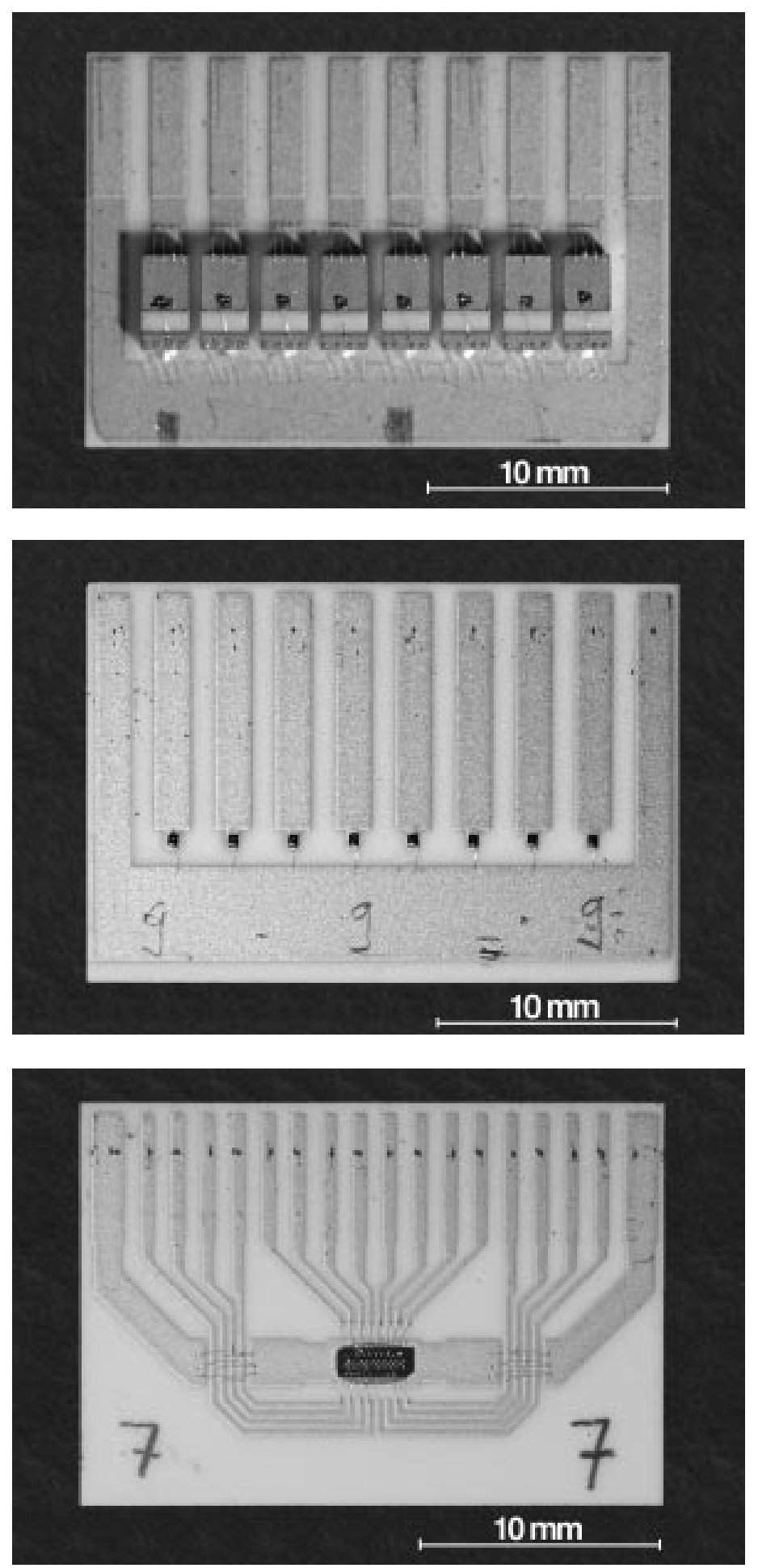

Figure 9: Devices mounted onto the ceramic boards used in the scanning machine. Top: eight ABB LEDs with micro lenses (dark spots); middle: eight GEC LEDs; bottom: one twenty-fold VCSEL array with 16 connector pads. 


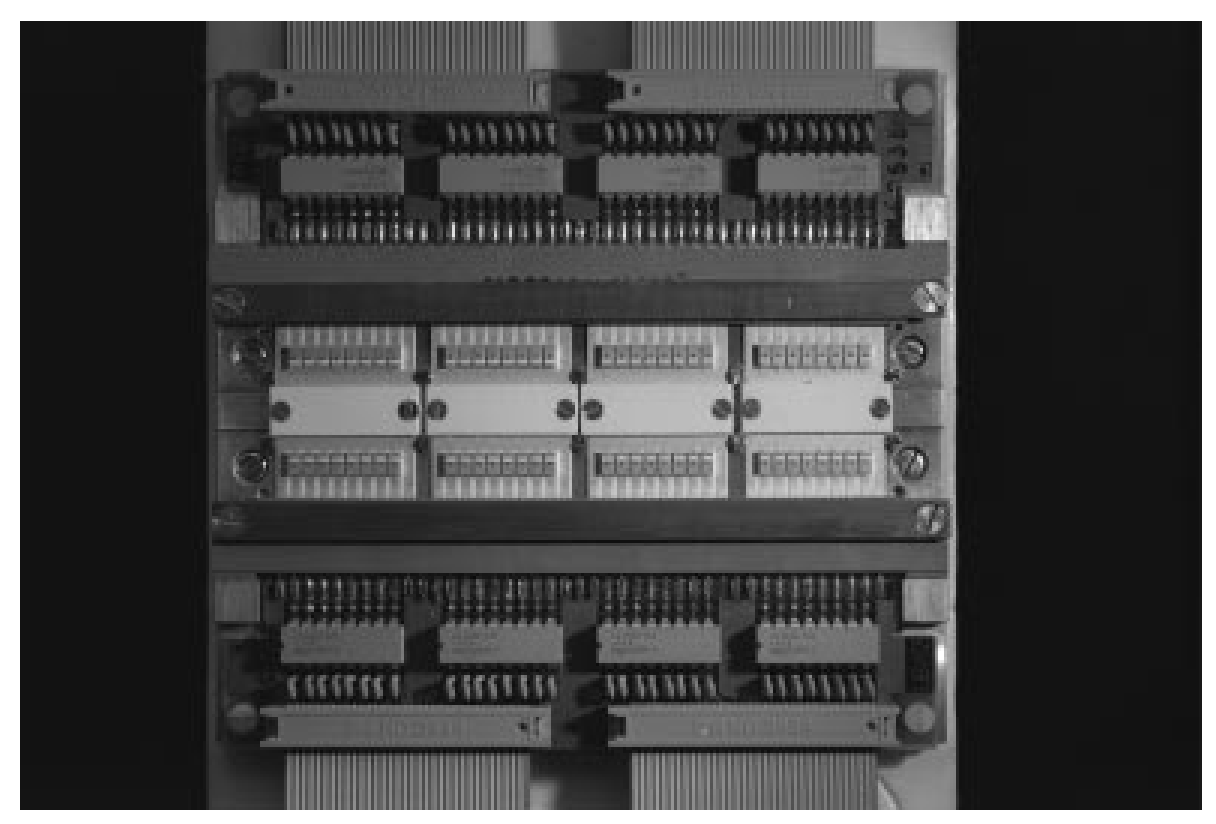

Figure 10: Temperature controlled support (block) with eight modules installed, each one carrying eight ABB LEDs

Each block has space and driver electronics for 64 devices. The LEDs are assembled as surface mounted devices in groups of 8 on ceramic boards (called modules), which ensure a good thermal contact with the temperature controlled blocks. The VCSELs are produced as 20-fold arrays which are mounted on the same kind of ceramic boards but providing 16 connector pads at half the pitch for an alternate operation of eight devices. Electrical contacts are made by wire-bonding from the diodes to the modules, and by special connectors from the modules to signal terminating boards, and from there via flat ribbon cables to the driver boards located outside of the scanning machine. Thus for the irradiation the modules could be easily removed from and reinserted into the machine.

The operating current of the LEDs or VCSELs can be set independently for each module between $0 \mathrm{~mA}$ and $100 \mathrm{~mA}$, and each device can be individually switched on, off, or set to pulsed mode. For each diode the current and the forward voltage, the light output (including the spatial profile of the emitted light cone), and the fall and rise time can be measured automatically under computer control, using a digital oscilloscope (LeCroy 9450) with a bandwidth of $350 \mathrm{MHz}$ and a VME based 16-bit ADC card. Additional measurements were made of the temperature of each block, of the humidity inside the scanning machine, and of the temperature of the PIN diodes.

The operation of the scanning machine is controlled by a data acquisition system running on a dedicated OS/9 computer, allowing completely automated long term measurements. The data acquisition software was written in $\mathrm{C}++$ based on an object-oriented design, where much emphasis was put on reliable long-term operation and comprehensive logging of all measurements and of the machine state. 


\subsection{Normalization and Calibration of the Light Output Measurements}

For practical reasons the light output of the LEDs was usually measured at the actual operating temperature and forward current during the annealing and the aging. However, normalization to the default operating conditions (see table 3) was possible by measuring for each device the light output as function of the forward current and the temperature. Since the dependence of the light output from the operating current of LEDs changes during irradiation and annealing, measurements have been made before the irradiation and during the annealing when necessary. The normalized light output thus obtained can be directly related to the pre-irradiation measurements made at the default operating conditions in order to calculate the relative light output (RLO). For both types of LEDs the normalization procedure works well, and the errors introduced by the normalization are on the order of a few percent.

For VCSELs, however, a similar normalization procedure is not practical due to the strong and more complicated temperature dependence of the lasing threshold current, which would result in much larger errors of the normalization procedure. Both for VCSELs and for LEDs, only measurements made at the default operating conditions have been used for producing the histograms and the statistics reported thereafter in order to avoid normalization errors.

The measurement of the light output with the PIN diode inside the scanning machine results in a voltage signal which must be calibrated to obtain the optical power coupled into a multimode fibre. For this purpose, a few devices of each type were operated on a test bench and manually coupled to a short piece of 50/125 multimode fibre. The fibres were aligned in three axis to find the maximum light output, which was measured at the end of the fibre with a calibrated optical head. The LEDs were equipped with a micro-lens. Therefore, the maximum light output was found at a short distance from the LED. For VCSELs Butt coupling, i.e. the fibre is in direct contact with the emitter, was used, and a slight pressure was applied in order to avoid modal noise resulting from back reflection from the cleaved end of the fibre into the laser cavity. Not surprisingly, for VCSELs the light coupling into fibres was found to be very sensitive to the pressure applied, and therefore only an approximate calibration constant could be determined.

For the GEC LEDs, the optical power measured at the end of the actively aligned fibre was compared to the optical power obtained from packaged GEC LEDs with passively aligned fibres. The latter was in average $20 \%$ lower which was attributed to the different alignment technique. Since for the SCT the light output of the packaged devices is the relevant quantity, all calibration factors for the scanning machine were corrected for this $20 \%$ loss due to passive alignment.

\subsection{Irradiation}

Most of the LEDs and VCSELs were irradiated with either neutrons or protons, reaching fluences as high as $5 \cdot 10^{14} \mathrm{n} / \mathrm{cm}^{2}$ and $4 \cdot 10^{14} \mathrm{p} / \mathrm{cm}^{2}$, respectively. A few 


\begin{tabular}{|c|c|c|c|}
\hline $\begin{array}{c}\text { No. of } \\
\text { devices }\end{array}$ & Device type & $\begin{array}{c}\text { Operation mode } \\
\text { during irradiation }\end{array}$ & $\begin{array}{c}\text { Fluence } \\
\left(10^{14} \mathrm{p} / \mathrm{cm}^{2}\right)\end{array}$ \\
\hline \hline 35 & ABB LEDs & off & $\sim 0.1-1.6$ \\
4 & ABB LEDs & DC $(10 \mathrm{~mA})$ & $\sim 0.7-1.3$ \\
26 & ABB LEDs & pulsed $(10 \mathrm{~mA})$ & $\sim 1.4-2.2$ \\
\hline 26 & GEC LEDs & pulsed $(18 \mathrm{~mA})$ & $\sim 1.7-2.6$ \\
25 & GEC LEDs & off & $\sim 1.7-2.6$ \\
7 & GEC LEDs & pulsed $(20 \mathrm{~mA})$ & $\sim 1.2-2.2$ \\
7 & GEC LEDs & off & $\sim 1.2-2.2$ \\
7 & GEC LEDs & pulsed $(20 \mathrm{~mA})$ & $\sim 2.5-4.8$ \\
7 & GEC LEDs & off & $\sim 2.5-4.8$ \\
\hline 16 & VCSELs Ia & off & $2.1 \pm 0.2$ \\
\hline 19 & VCSELs Ib & pulsed $(4 \mathrm{~mA})$ & $1.8 \pm 0.2$ \\
17 & VCSELs Ib & off & $1.8 \pm 0.2$ \\
15 & VCSELs Ib & pulsed $(4 \mathrm{~mA})$ & $3.7 \pm 0.3$ \\
15 & VCSELs Ib & off & $3.7 \pm 0.3$ \\
\hline 3 & VCSELs II & pulsed $(2 \mathrm{~mA})$ & $3.7 \pm 0.3$ \\
4 & VCSELs II & off & $3.7 \pm 0.3$ \\
\hline
\end{tabular}

Table 4: Overview of proton irradiated LEDs and VCSELs, of the operating conditions during the irradiation, and of the fluences reached. All devices listed in the table were irradiated with $24 \mathrm{GeV}$ protons at the CERN PS. The error on the dosimetry is about $10 \%$ and is mainly due to uncertainties of the beam profile.

devices were reirradiated with neutrons after a first irradiation with neutrons or protons in order to check if there is any difference in the final relative light output if the irradiation is applied all at once or in two steps with an intermediate annealing treatment (see section 5.5). All irradiations were done at ambient temperature and no measurements were made during the irradiation ${ }^{8}$.

During the neutron irradiation, all devices were unbiased and open circuited. About half of the devices irradiated with protons were operated in a pulsed mode with a duty cycle of $25 \%$ at approximately the default operating current. Four ABB LEDs were biased with a DC forward current of $10 \mathrm{~mA}$. The remaining devices were off and open circuited during proton irradiation. Tables $4-6$ give an overview of the devices irradiated, of the operating conditions during the irradiation, and of the fluences reached.

Most of the neutron irradiation took place at the ISIS facility at the Rutherford Appleton Laboratory (RAL) [76], where neutrons with an energy spectrum peaking at about $1 \mathrm{MeV}$ are available from a spallation source. The neutron energy spectrum

\footnotetext{
${ }^{8}$ In complementary irradiation studies done at the University of Birmingham, a few GEC LEDs were irradiated at about $-7^{\circ} \mathrm{C}$ without observing any significant difference in the resulting radiation damage [75]. The light output of LEDs during the irradiation was measured previously by several groups $[6,8,56]$.
} 


\begin{tabular}{|c|c|c|c|}
\hline $\begin{array}{c}\text { No. of } \\
\text { devices }\end{array}$ & \multirow{2}{*}{ Device type } & \multicolumn{2}{|c|}{ Fluence $\left(10^{14} \mathrm{n} / \mathrm{cm}^{2}\right)$} \\
& & $>\mathrm{keV}$ & $<10 \mathrm{keV}$ \\
\hline \hline 15 & ABB LEDs & $0.85 \pm 0.12$ & $26.9 \pm 0.5$ \\
23 & ABB LEDs & $3.2 \pm 0.5$ & $42.1 \pm 0.7$ \\
16 & ABB LEDs & $5.3 \pm 1.8$ & $33.9 \pm 0.6$ \\
\hline 29 & GEC LEDs & $0.77 \pm 0.12$ & $15.3 \pm 0.3$ \\
27 & GEC LEDs & $4.4 \pm 0.5$ & $19.2 \pm 0.4$ \\
\hline 47 & VCSELs Ib & $0.77 \pm 0.12$ & $15.3 \pm 0.3$ \\
31 & VCSELs Ib & $4.4 \pm 0.5$ & $19.2 \pm 0.4$ \\
\hline 14 & VCSELs II & $4.4 \pm 0.5$ & $19.2 \pm 0.4$ \\
\hline
\end{tabular}

Table 5: Overview of neutron irradiated LEDs and VCSELs and of the fluences reached. All devices listed in the table were irradiated with $\sim 1 \mathrm{MeV}$ neutrons at the RAL ISIS facility, where the dosimetry was done separately for the $\sim 1 \mathrm{MeV}(E>10 \mathrm{keV})$ and for the thermal background neutrons $(E<10 \mathrm{keV})$. Since the contribution of thermal neutrons to the displacement damage in GaAs is small compared to the one of $\sim 1 \mathrm{MeV}$ neutrons, the thermal neutron fluence was neglected (see text). All devices were not operated during the irradiation.

\begin{tabular}{|c|c|c|r|c|}
\hline $\begin{array}{c}\text { No. of } \\
\text { devices }\end{array}$ & Device type & Irradiation & \multicolumn{2}{|c|}{ Fluence $\left(10^{14} \mathrm{n} / \mathrm{cm}^{2}\right)$} \\
facility & $>10 \mathrm{keV}$ & $<10 \mathrm{keV}$ \\
\hline \hline 8 & ABB LEDs & Dynamitron & $2.5-3.2$ & $\mathrm{n} / \mathrm{a}$ \\
\hline 8 & GEC LEDs & Dynamitron & $2.1-2.7$ & $\mathrm{n} / \mathrm{a}$ \\
8 & GEC LEDs & RAL ISIS & $10.5 \pm 1.4$ & $30.7 \pm 0.5$ \\
\hline
\end{tabular}

Table 6: Overview of reirradiated LEDs and VCSELs and of the fluence reached 


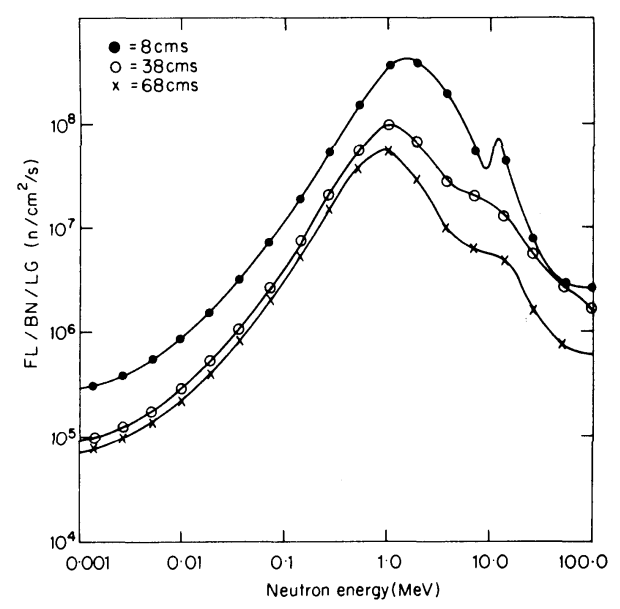

Figure 11: Neutron energy spectrum at the RAL ISIS facility, for three different distances from the target [76]

at ISIS is shown in figure 11 . The $\gamma / \mathrm{n}$ and the $\mathrm{p} / \mathrm{n}$ fluence ratios are about $10 \%$ and $10^{-3}$, respectively [77]. The neutron flux is rather low, varying between about $1.5 \cdot 10^{12}$ and $4.3 \cdot 10^{13} \mathrm{n} \mathrm{cm}^{-2}$ day $^{-1}$ depending on the operating conditions of the proton synchrotron and the position of the samples. In addition to the $\sim 1 \mathrm{MeV}$ neutrons, there is a large background of thermal neutrons which, according to NIEL calculations [45], cause about two orders of magnitude less displacement damage in GaAs than $1 \mathrm{MeV}$ neutrons. For most of the neutron irradiations done at ISIS, the contribution of the thermal neutrons to the total displacement damage is estimated to be less than $\sim 5 \%$, and, in the case of the devices irradiated with the most unfavorable ratio of fast to thermal neutrons, less than $10 \%$. Given an uncertainty of the dosimetry of typically $15 \%$, the contribution of the thermal neutron background was neglected.

In addition to the neutron irradiation at ISIS, for the reirradiation studies a few devices were irradiated at the Dynamitron in Birmingham [78], where neutrons are produced by deuterons hitting a $\sim 0.75 \mathrm{~mm}$ thick beryllium metal target. The energy of the incident deuterons can be adjusted in the range from 2.6 to $7.0 \mathrm{MeV}$. The resulting neutron energy spectrum is shown in figure 12. Very high fluxes up to approximately $8 \cdot 10^{14} \mathrm{n} \mathrm{cm}^{-2}$ day $^{-1}$ can be achieved [79].

All proton irradiations were done at CERN, where $24 \mathrm{GeV}$ protons are available from the CERN PS (proton synchrotron) and fluences of approximately $10^{14} \mathrm{p} \mathrm{cm}^{-2}$ day $^{-1}$ can be reached. Due to the extraction of the protons from the PS, they are delivered in bunches of $\sim 10^{11}$ protons every 14 seconds. During the irradiation the modules with the LEDs or VCSELs were mounted one behind the other in slide holders aligned with the beam axis, usually behind devices irradiated by other groups. Because of the rather large size of about $2 \mathrm{~cm}$ of the modules in the transverse direction, the beam was not always uniform over the whole module, making the dosimetry with aluminum foils difficult and leading to higher uncertainties in the dosimetry than usual for irradiation at the PS. 


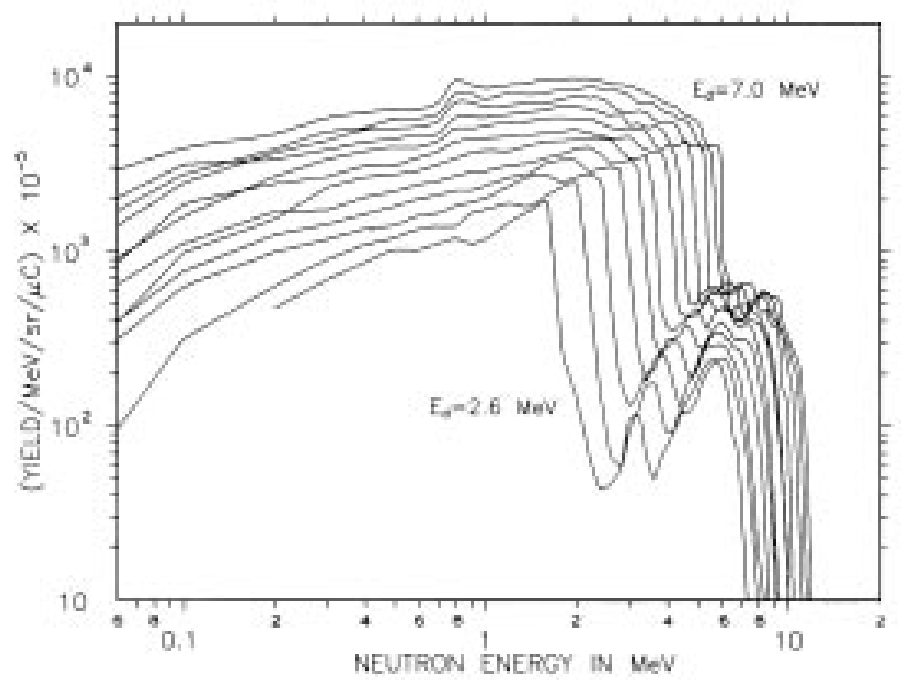

Figure 12: ${ }^{9} \mathrm{Be}(\mathrm{d}, \mathrm{n})$ thick target spectrum for deuteron energies of 2.6 to $7 \mathrm{MeV}$ in steps of $0.4 \mathrm{MeV}[78]$

After the irradiation period of typically a few days for the proton irradiation and several weeks for the neutron irradiation, the irradiated devices were stored at room temperature until their activity had decayed to a level allowing the shipping of the devices back to the laboratory.

\subsection{Measurement Procedure for Irradiated Devices}

After shipping the irradiated devices back to the laboratory, they were reinstalled into the scanning machine and cooled down to either $+10^{\circ} \mathrm{C}$ or $-10^{\circ} \mathrm{C}$ for the annealing treatment. The first measurement of all irradiated devices was done at the default forward current given in table 3. In the case of VCSELs, for some (but not all) devices on each array, light output vs. current and forward voltage vs. current curves were measured, too.

Once the first measurement after irradiation was made, the forward current was set to the desired annealing current and the devices were operated in DC mode for several days or weeks and frequently measured in order to monitor the progress of the annealing. When no further annealing was observed at a given forward current, the annealing treatment was either stopped or continued at a higher current in order to investigate the dependence of the injection annealing on the forward current.

In order to study the long term reliability of irradiated LEDs and VCSELs, the aging was accelerated by operating the diodes at an elevated temperature of $50^{\circ} \mathrm{C}$ for several months once the annealing was finished. However, for some of the GEC LEDs the annealing was extremely slow at the chosen forward current and would have taken many months to finish. Therefore, before the annealing was finished, these devices were set at $50^{\circ} \mathrm{C}$ where the annealing was continued at the elevated annealing current. For all other devices the long term studies at $50^{\circ} \mathrm{C}$ were performed at the default operating current. 


\section{Results}

\subsection{Overview}

In the following sections we describe the results obtained in our studies. In section 5.2 we present the damage constants obtained for GEC LEDs from the first measurements made after irradiation. The annealing behavior of LEDs and VCSELs is described in section 5.3 and 5.4, respectively, followed by the results of the reirradiation studies in section 5.5. The long term reliability is discussed in section 5.6 (life time) and 5.7 (estimated relative light output after 10 years of operation at LHC). The overall statistics are presented in section 5.8 and the behavior of failing devices is described in more detail in section 5.9.

\subsection{Damage Constants for GEC LEDs}

By measuring the light output after irradiation, LEDs may be used to measure displacement damage. As shown in section 2.5, the relative light output after irradiation with a fluence $\phi$ can be related to the damage constant $K$, or, equivalently, to $k \equiv \tau_{0} K$ :

$$
\left(\frac{1}{R L O}\right)^{\frac{2}{3}}=1+\tau_{0} K \phi
$$

In order to determine a physically meaningful value of $k$, it is important that either no injection annealing takes place, or that the amount of annealing can be quantified, since the defect concentration and thus the damage constant changes during the annealing ${ }^{9}$.

Due to the fact, that the GEC LEDs exhibit no or only a very slow annealing at the default current of $20 \mathrm{~mA}$, (almost) no injection annealing takes place during the few ms of operation required for the light output measurement. Therefore, those GEC LEDs that were not operated during the irradiation can be used to determine the parameter $k$ both for neutron $\left(k_{n}\right)$ and for proton $\left(k_{p}\right)$ irradiation. According to the NIEL hypothesis the ratio $k_{p} / k_{n}$ is determined only by the ratio of the proton and neutron NIEL, and therefore a measurement of $k_{p} / k_{n}$ provides a direct test of the NIEL hypothesis and of the theoretical NIEL calculations.

Damage constant fits for both neutron and proton irradiation of GEC LEDs are shown in figure 13. The resulting proton-to-neutron damage ratio $k_{p} / k_{n}=3.2 \pm 0.1$ for $24 \mathrm{GeV}$ protons and ISIS neutrons is in excellent agreement with the NIEL calculations for GaAs summarized in table 1 (in section 2.4) giving a ratio of 3.2. This result provides an important confirmation of the NIEL hypothesis, which is crucial for the extrapolation of radiation hardness studies to the LHC environment.

\footnotetext{
${ }^{9}$ The damage constant $K$ could be determined more directly by measuring the minority carrier life time in the frequency domain. This can be achieved by modulating a DC bias current with an $\mathrm{AC}$ signal and by measuring the $\mathrm{AC}$ frequency response [55]. Since such measurements can be made at low currents, the influence of injection annealing can be minimized. However, the electronics of the scanning machine does not support this technique, and direct measurements of the fall time of light signals turned out to be nearly impossible given the very low light output of LEDs after irradiation.
} 

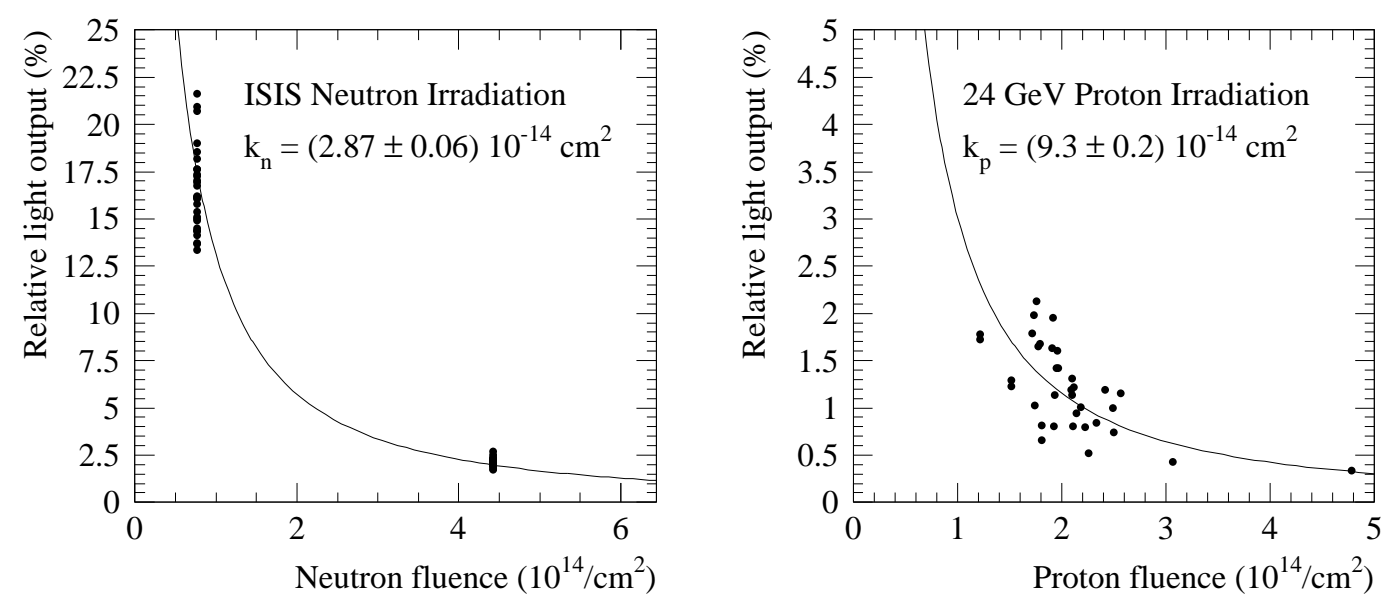

Figure 13: Damage function fit for GEC LEDs irradiated with ISIS neutrons (left) and $24 \mathrm{GeV}$ protons (right). The errors are the statistical errors of the fitting procedure, assuming an uncertainty of $15 \%(10 \%)$ in the dosimetry of the neutron (proton) irradiation, and an error of $5 \%$ for the light output measurements.

If a similar fit is done for GEC LEDs which were pulsed with a $20 \mathrm{~mA}$ forward current during the irradiation, a lower value of $\tilde{k_{p}}=(6.8 \pm 0.2) \cdot 10^{-14} \mathrm{~cm}^{2}$ is found for proton irradiation. As expected, the damage constant is significantly lower due to the injection annealing already taking place during the irradiation.

\subsection{Annealing of LEDs}

\subsubsection{Injection Annealing of ABB Hafo LEDs}

A typical behavior of an ABB LED that was not biased during the irradiation is shown in figure 14a: After an irradiation with $1.6 \cdot 10^{14} \mathrm{p} / \mathrm{cm}^{2}$, the relative light output (see section 3.2.1) of this particular LED was decreased to a few percent. The operation of this LED at $10 \mathrm{~mA}$ and $+10^{\circ} \mathrm{C}$ for about 12 days did not change the light output significantly. However, when the forward current was increased to $50 \mathrm{~mA}$, a fast annealing occured, increasing the relative light output to about $40 \%$ within a few hours.

A different behavior is shown in figure $14 \mathrm{~b}$ for another ABB LED irradiated with $3.2 \cdot 10^{14} \mathrm{n} / \mathrm{cm}^{2}$. In this case, annealing took already place at a current of $10 \mathrm{~mA}$ (and at $+10^{\circ} \mathrm{C}$ ), raising the relative light output from $\sim 10 \%$ to $\sim 60 \%$. However, when the operating current was increased from $10 \mathrm{~mA}$ to $30 \mathrm{~mA}$ after 6 days, antiannealing was observed which decreased the relative light output to $40 \%$.

Both annealing and anti-annealing have been observed both after proton and after neutron irradiation. Anti-annealing of ABB LEDs has been seen at different forward currents between $10 \mathrm{~mA}-50 \mathrm{~mA}$, although it was more pronounced at higher currents and not all devices showed the same amount of anti-annealing. 

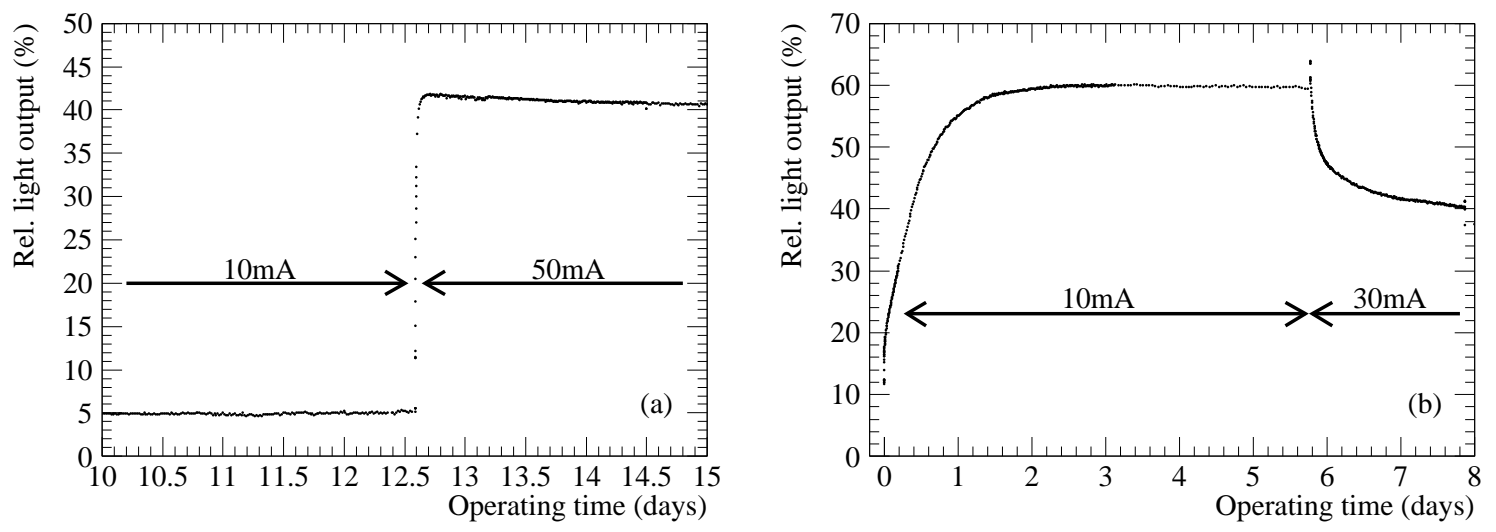

Figure 14: Typical annealing behavior of ABB LEDs. a) Relative light output of an ABB LED after irradiation with $1.6 \cdot 10^{14} \mathrm{p} / \mathrm{cm}^{2}$ as a function of DC operating time at $+10^{\circ} \mathrm{C}$. Little annealing occurs at $10 \mathrm{~mA}$, but an increase of the forward current to $50 \mathrm{~mA}$ results in a fast annealing. b) Relative light output of an ABB LED after irradiation with $3.2 \cdot 10^{14} \mathrm{n} / \mathrm{cm}^{2}$, as a function of $\mathrm{DC}$ operating time at $+10^{\circ} \mathrm{C}$. Increasing the current from $10 \mathrm{~mA}$ to $30 \mathrm{~mA}$ does not produce further annealing but results in antiannealing.

While the behavior described above can be understood qualitatively as being due to diffusion processes involving different types of defects in the LED junction, a quantitative modeling would require microscopic information about the defect types involved, their electrical properties and their concentrations in the LED junction. Such information could be obtained e.g. from Deep Level Transient Spectroscopy (DLTS) measurements [58, 80].

\subsubsection{Injection Annealing of GEC-Marconi LEDs}

The annealing behavior of GEC LEDs is less favorable than the one of ABB LEDs. At currents below $40 \mathrm{~mA}$ there is almost no or only a very slow annealing (figure 15a). An annealing current of $40 \mathrm{~mA}-80 \mathrm{~mA}$ is required to produce a significant amount of annealing. While at $40 \mathrm{~mA}$ the annealing process is rather slow (figure 15b), there seems to be little difference in the annealing at currents between $50 \mathrm{~mA}-80 \mathrm{~mA}$. Figure $15 \mathrm{c}$ shows a GEC LED irradiated with $4.4 \cdot 10^{14} \mathrm{n} / \mathrm{cm}^{2}$, which annealed within a few days at $50 \mathrm{~mA}$ and $-10^{\circ} \mathrm{C}$. At currents higher than $80 \mathrm{~mA}$, anti-annealing was observed even for unirradiated devices (figure 15d).

\subsubsection{Temperature Dependence of the Annealing}

All devices were at room temperature during irradiation, while being stored after the irradiation, and during the transport back to the laboratory. However, in complementary irradiation studies done at the University of Birmingham, a few GEC LEDs were irradiated at about $-7^{\circ} \mathrm{C}$ without observing any significant difference in the resulting radiation damage [75]. Further, except for a yet unexplained drop 

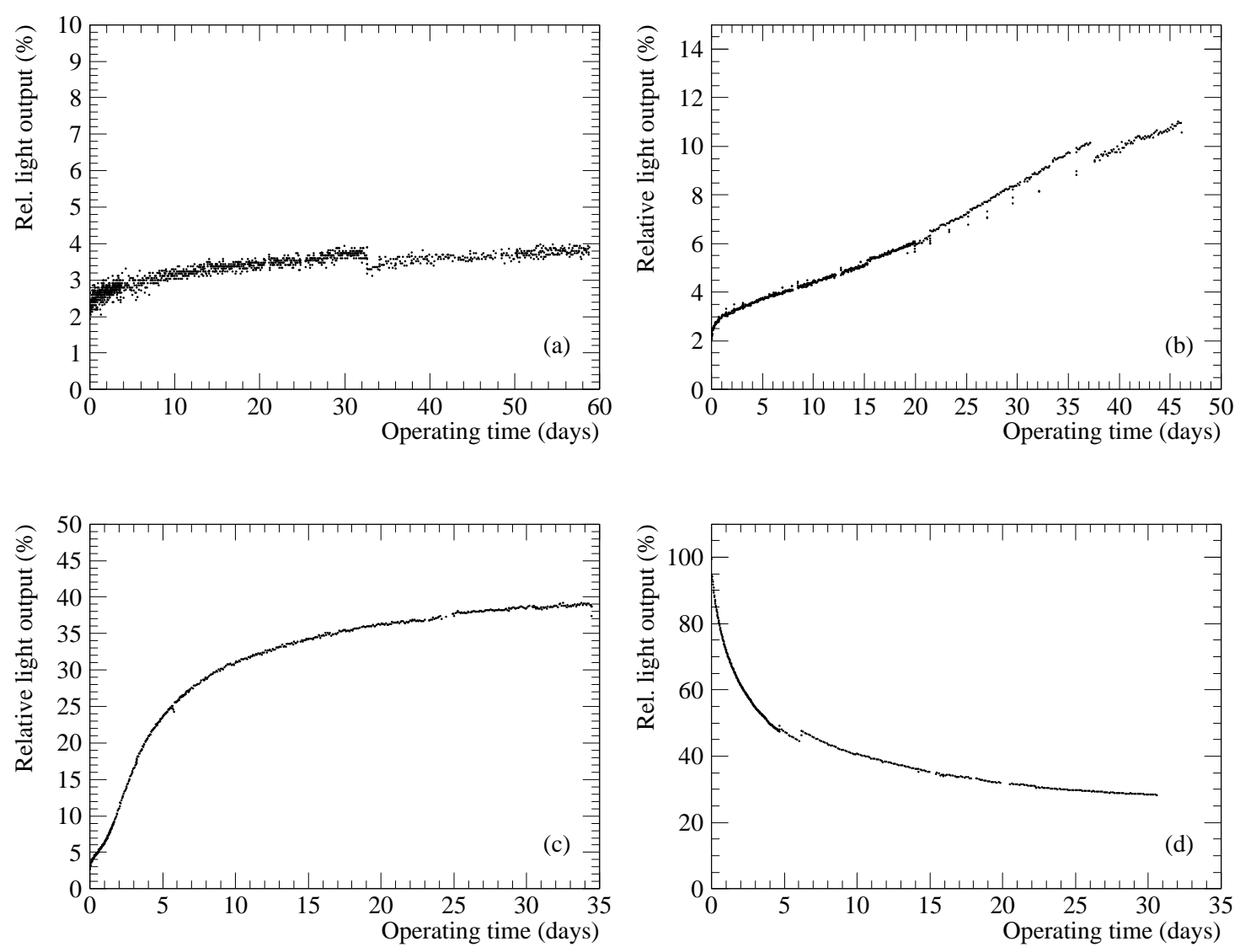

Figure 15: Typical annealing behavior of GEC LEDs. Relative light output of a GEC LED a) at $20 \mathrm{~mA}$ and $10^{\circ} \mathrm{C}$ after irradiation with $2.1 \cdot 10^{14} \mathrm{p} / \mathrm{cm}^{2}$, b) at $40 \mathrm{~mA}$ and $10^{\circ} \mathrm{C}$ after irradiation with $2.2 \cdot 10^{14} \mathrm{p} / \mathrm{cm}^{2}, \mathrm{c}$ ) at $50 \mathrm{~mA}$ and $-10^{\circ} \mathrm{C}$ after irradiation with $4.4 \cdot 10^{14} \mathrm{n} / \mathrm{cm}^{2}$, d) at $100 \mathrm{~mA}$ anti-annealing is observed even without irradiation. See text.

in light output for a single ABB LED (see section 5.9) no significant annealing or anti-annealing was observed during room temperature storage periods in the laboratory. As mentioned in section 2.6, thermal annealing is not expected at room temperature for LEDs [37, 55].

Most of the annealing studies were carried out at either $+10^{\circ} \mathrm{C}$ or $-10^{\circ} \mathrm{C}$. Comparing the annealing behavior of devices irradiated with the same fluence and annealed at the same forward current but at a different temperature of either $+10^{\circ} \mathrm{C}$ or $-10^{\circ} \mathrm{C}$, no significant difference was found in the final relative light output at the end of the annealing treatment.

However, in a few cases the annealing of LEDs was accelerated when the operating temperature was increased from $\pm 10^{\circ} \mathrm{C}$ to $50^{\circ} \mathrm{C}$ in order to accelerate the aging. For most of the ABB LEDs, the annealing was completely finished when the high temperature operation was started. Therefore, only a slight or no additional annealing was observed at $50^{\circ} \mathrm{C}$. The only exception is shown in figure $16 \mathrm{a}$. This 

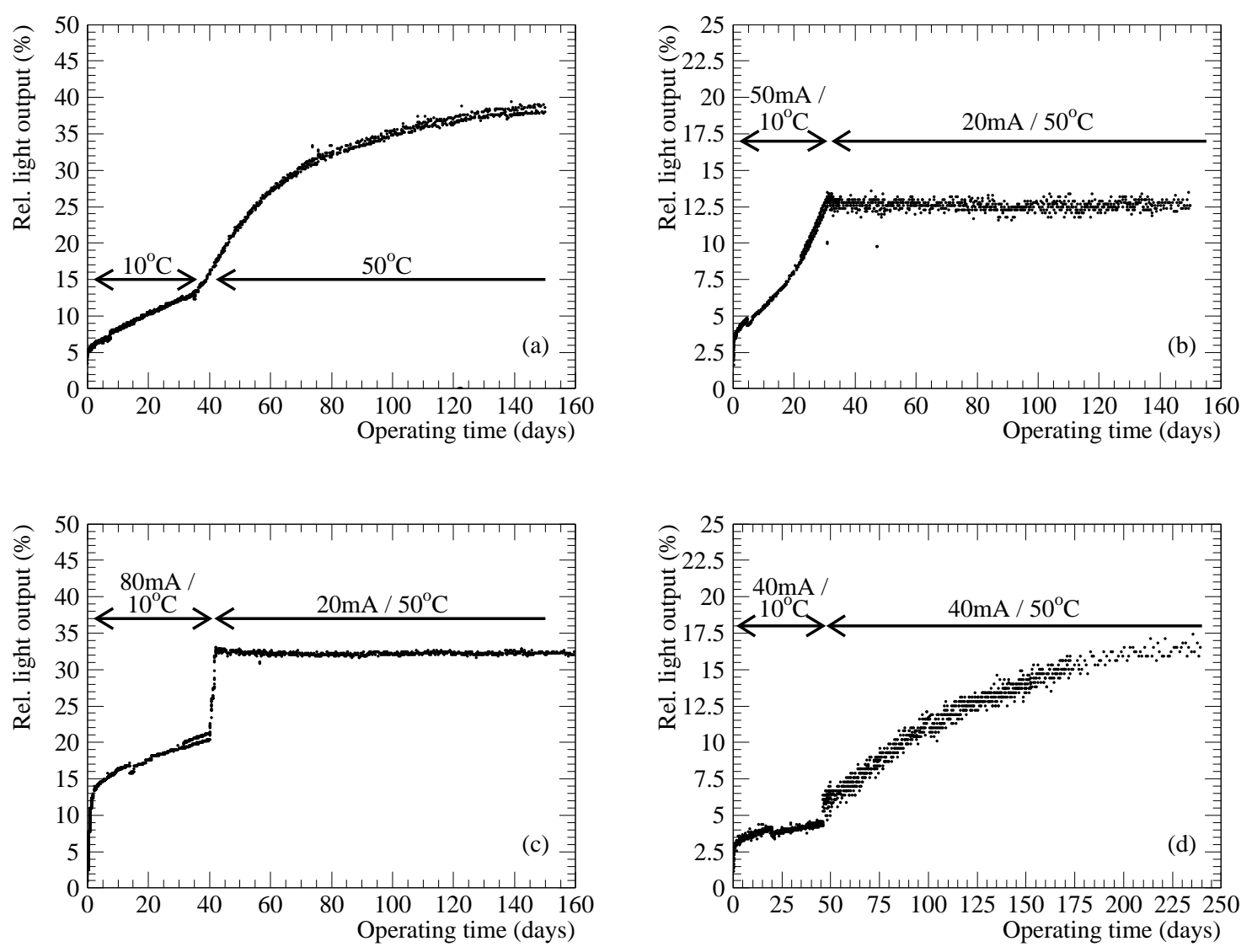

Figure 16: Temperature dependence of the annealing: a) ABB LED irradiated with $5.3 \cdot 10^{14} \mathrm{n} / \mathrm{cm}^{2}$ showing an acceleration of the annealing at $20 \mathrm{~mA}$ when the temperature was raised; b) most of the GEC LEDs stopped the annealing when the forward current was decreased to $20 \mathrm{~mA}$; c) example of a GEC LED annealing quickly at elevated temperature but low forward current; d) the annealing of a GEC LED at $40 \mathrm{~mA}$ was accelerated when the temperature was increased to $50^{\circ} \mathrm{C}$.

ABB LED showed a rather slow annealing after the irradiation with $5.3 \cdot 10^{14} \mathrm{n} / \mathrm{cm}^{2}$. When the temperature was raised, the annealing was accelerated and reached a relative light output of $40 \%$, which is in good agreement with the other LEDs on the same module that annealed completely before the operating temperature was increased.

However, for GEC LEDs the annealing takes much more time and for most of the devices the annealing was not completely finished before the temperature was raised to $50^{\circ} \mathrm{C}$. Therefore, the relative light output continued to increase during the high temperature operation. Obviously, the amount of annealing observed at $50^{\circ} \mathrm{C}$ depends on the forward current, too. If the forward current was reduced to the nominal $20 \mathrm{~mA}$, the annealing was usually stopped (figure 16b). A few exceptions have been observed like e.g. the GEC LED shown in figure $16 \mathrm{c}$ which exhibits a very strong annealing at $50^{\circ} \mathrm{C}$ and $20 \mathrm{~mA}$ after 40 days of annealing at $80 \mathrm{~mA}$. 
While in most cases the aging of the devices was studied at the default current (see table 3), some GEC LEDs (for which considerable more annealing was expected) were continued to be operated at an elevated forward current during the high temperature aging period. For these LEDs the annealing was accelerated at $50^{\circ} \mathrm{C}$ (figure 16d). However, the final relative light output when the annealing was finished does not differ from those devices completely annealed at $\pm 10^{\circ} \mathrm{C}$.

\subsubsection{Summary}

The results of the short term annealing studies of LEDs are summarized in figure 17 by plotting the relative light output of individual LEDs after irradiation as a function of the fluence. The fluences for the neutron irradiation (with $\sim 1 \mathrm{MeV}$ neutrons) at the RAL ISIS facility, and for the proton irradiation (with $24 \mathrm{GeV}$ protons) at the CERN PS, are given by the top and the bottom axis, respectively. Using the NIEL calculations summarized in table 1 , these axes were scaled such that the NIEL is the same for the corresponding neutron and proton fluences. Thus if the radiation damage in LEDs after the annealing can be parameterized by the NIEL, the relative light outputs after neutron and proton irradiation should concur in figure 17. Indeed, within the fairly large spread of the data, which is partly due to the uncertainties in the dosimetry, the proton and neutron irradiation results match fairly well.

The importance of injection annealing is evident from figure 17 when comparing the measurements of the relative light output before (dots) and after (large symbols) the annealing. A comparison of the ABB LEDs which were annealed with a maximum current of $30 \mathrm{~mA}$ (full circles) with those annealed at up to $50 \mathrm{~mA}$ (hollow circles), shows that the latter generally reach a higher relative light output. Thus, the degree of the annealing depends on the maximum annealing current applied.

If the LEDs are operated during the irradiation as will be the case at LHC, depending on the radiation flux and the operating current, some or all of the annealing will take place during the irradiation. Consequently, the light output measured shortly after the irradiation is much higher than if the LEDs are off during the irradiation. This behavior was observed for about one third of the LEDs which were operated in a pulsed mode during proton irradiation. For GEC LEDs which were biased during irradiation, a significantly lower damage constant was found (see section 5.2).

\subsection{Annealing of VCSELs}

The degradation of VCSELs by irradiation and the subsequent annealing is best discussed using light output vs. current plots. Figure 18 shows such plots for two VCSELs irradiated with $1.8 \cdot 10^{14} \mathrm{p} / \mathrm{cm}^{2}$. One was off during the irradiation (figure 18a) while the other was operated in pulsed mode with a current of $4 \mathrm{~mA}$ and a duty cycle of $\sim 25 \%$ (figure $18 \mathrm{~b}$ ). The first measurement after the irradiation (squares) reflects this fact: the un-biased one gives no light up to a forward current of $5 \mathrm{~mA}$ while the pulsed device shows very little degradation. After an annealing period of 5.9 days at $4 \mathrm{~mA}$ and $+10^{\circ} \mathrm{C}$ both VCSELs recovered almost completely. 


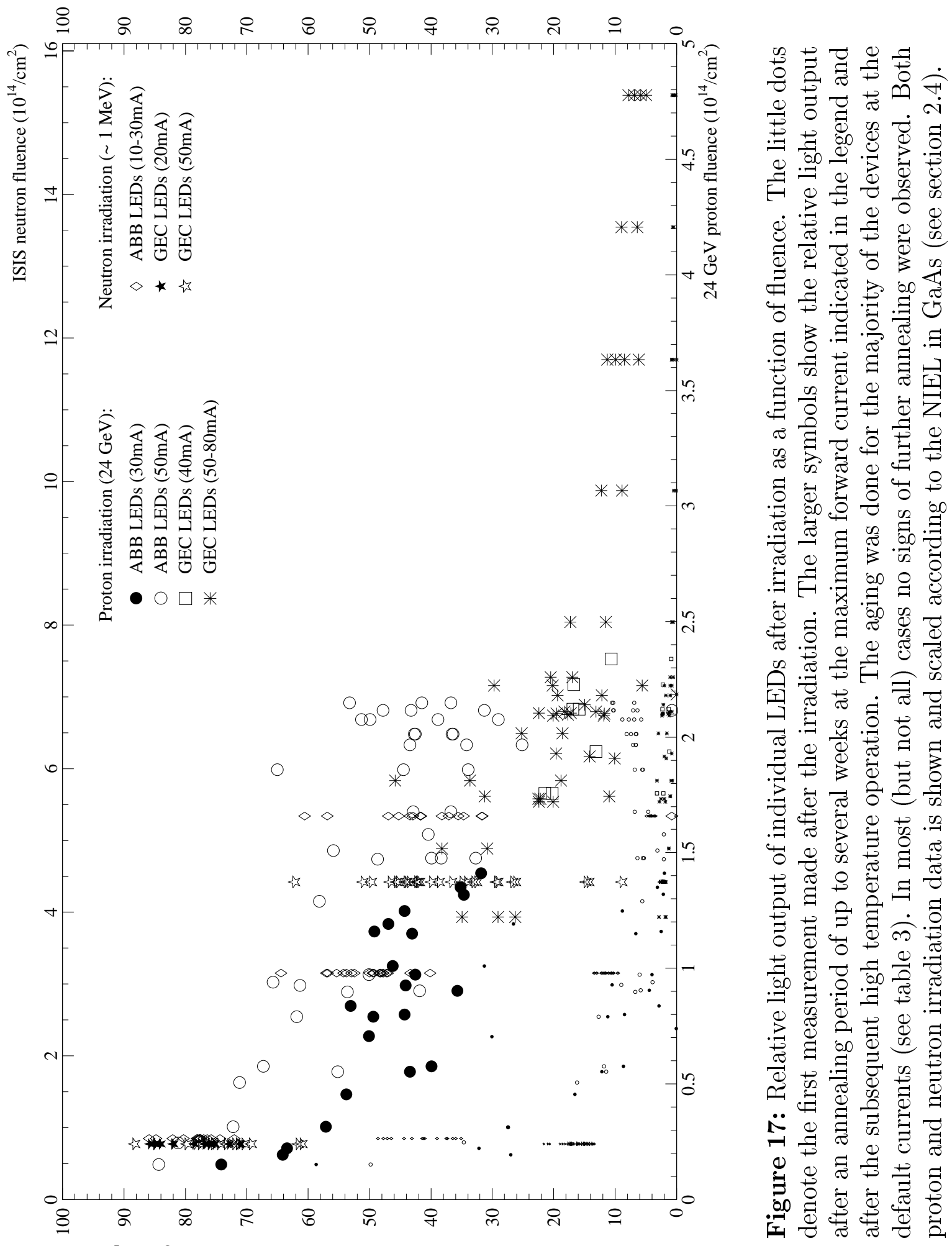

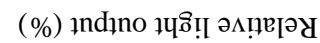



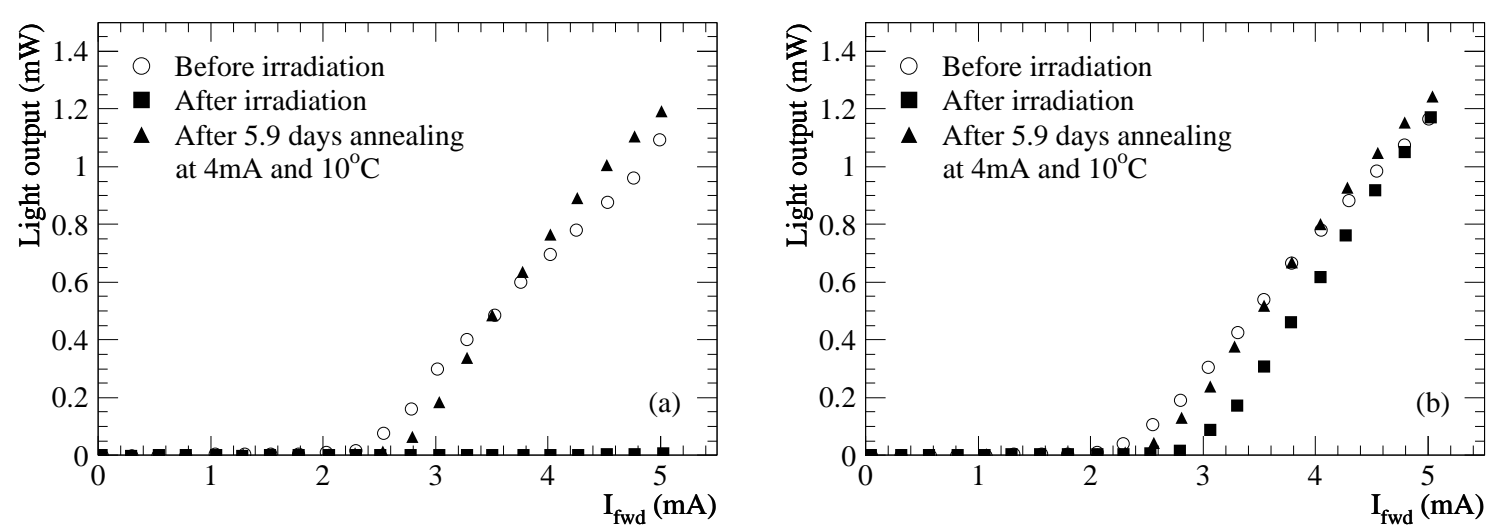

Figure 18: Light output vs. current before the irradiation, after the irradiation and after 5.9 days annealing shown for two VCSELs irradiated with $1.8 \cdot 10^{14} \mathrm{p} / \mathrm{cm}^{2}$ : a) VCSEL not operated during irradiation, b) VCSEL pulsed with $4 \mathrm{~mA}$ during irradiation

The outstanding radiation hardness of VCSELs is evident from figure 19. VCSELs irradiated with fluences up to $3.7 \cdot 10^{14} \mathrm{p} / \mathrm{cm}^{2}$ yield, after a short annealing period of a few hours to days, as much light as before the irradiation. For some devices, even a lower threshold current and a better light output than before the irradiation were observed. This behavior is not yet understood.

\subsection{Reirradiation Studies}

The irradiation of most devices used in this test was done in one step without intermediate annealing periods. This is an unrealistic scenario with respect to LHC where the total fluence is applied over ten years with long pauses in between. Therefore, a few devices have been irradiated twice with an intermediate annealing period in order to check if there is a significant different relative light output with respect to a single irradiation with the same total fluence.

Table 7 summarizes the irradiation and annealing conditions of the reirradiated devices. The total fluence of both irradiations is calculated by scaling the individual fluences with the NIEL in GaAs (see section 2.4). As shown in figure 20 the relative light output of the LEDs irradiated twice with a high total fluence tend to be somewhat higher than the LEDs having the total fluence applied at once. At lower fluences, i.e. at an equivalent fluence of about $2 \cdot 10^{14} \mathrm{p} / \mathrm{cm}^{2}$, no significant difference is found. 


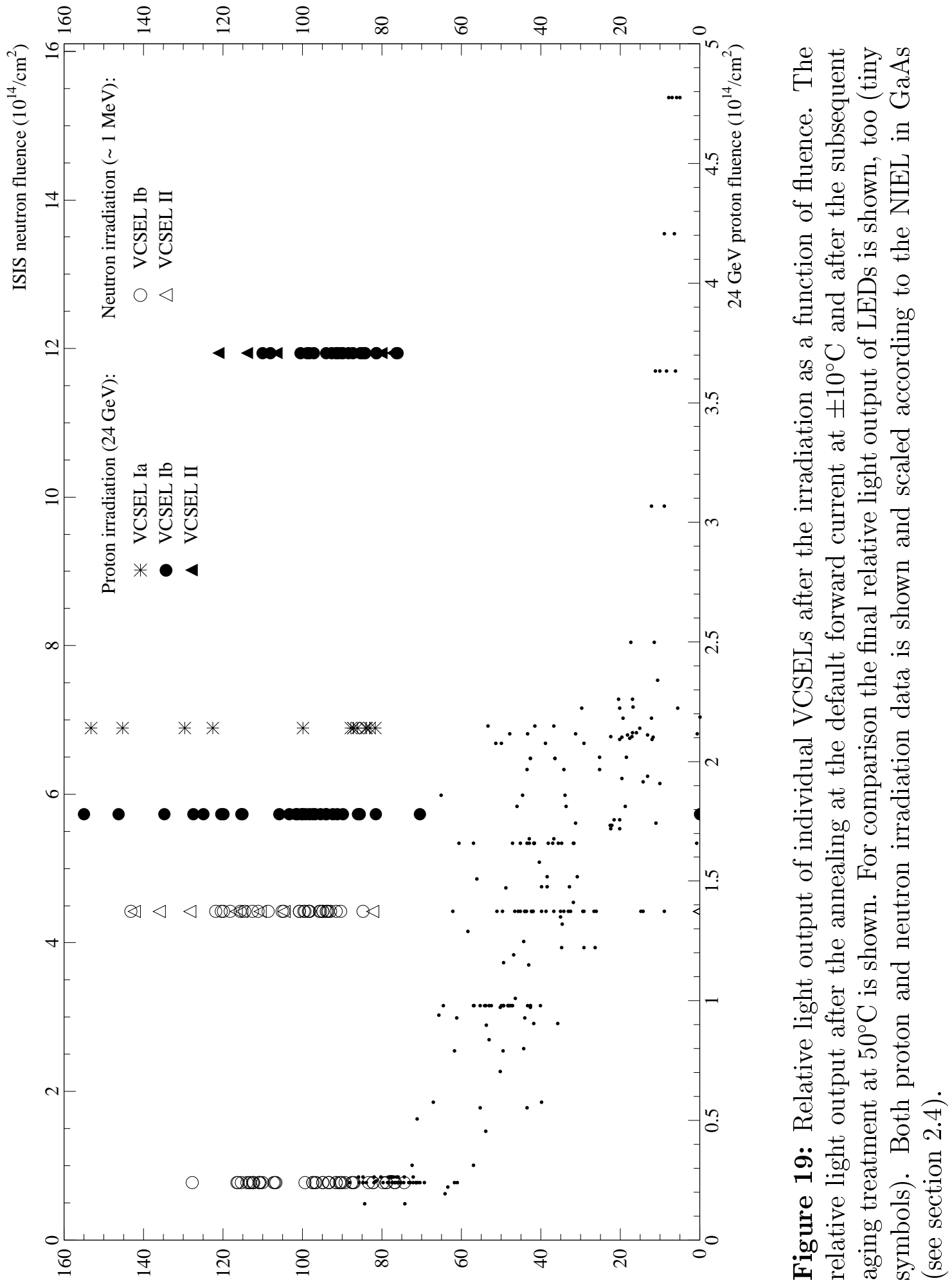

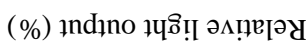




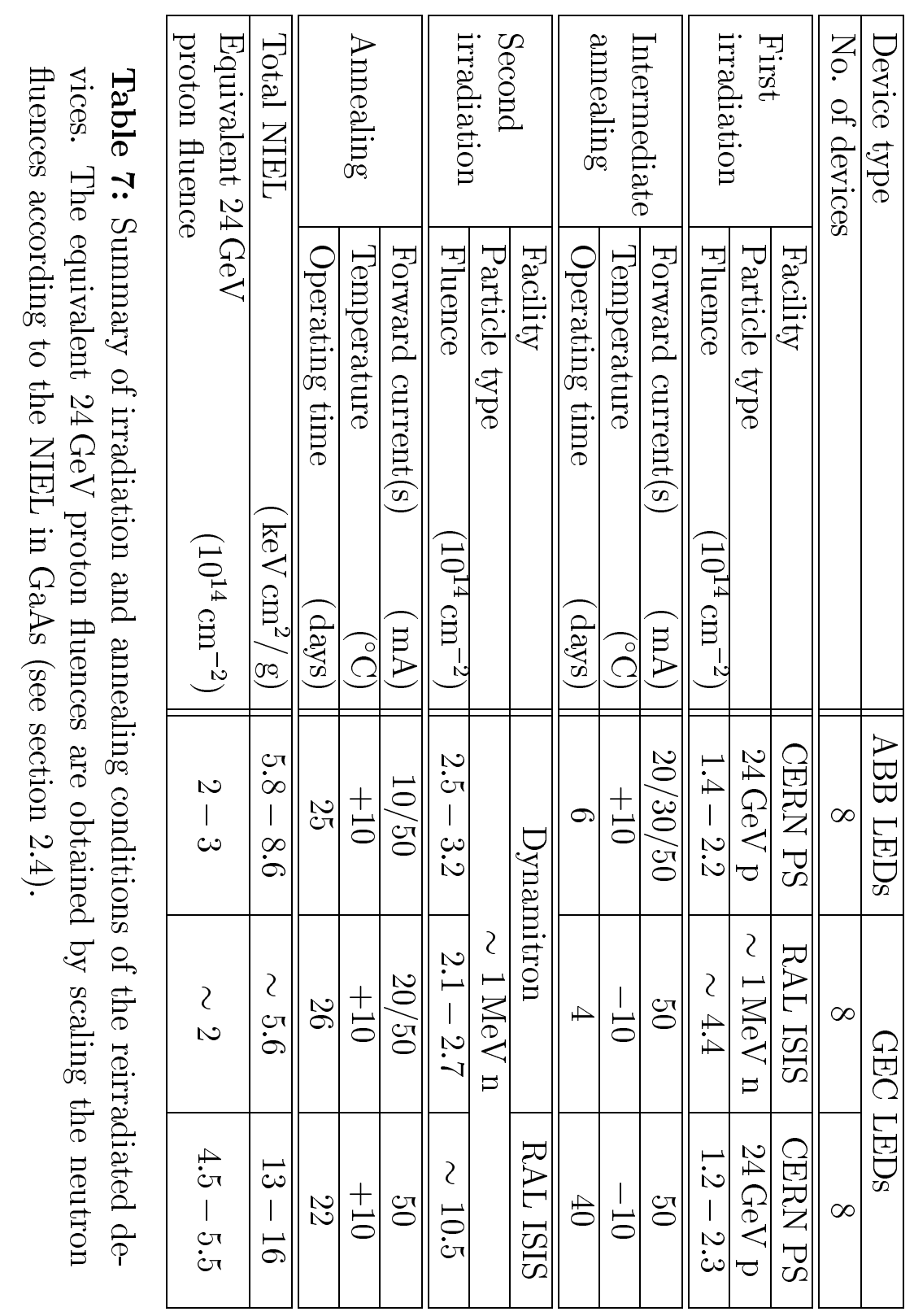




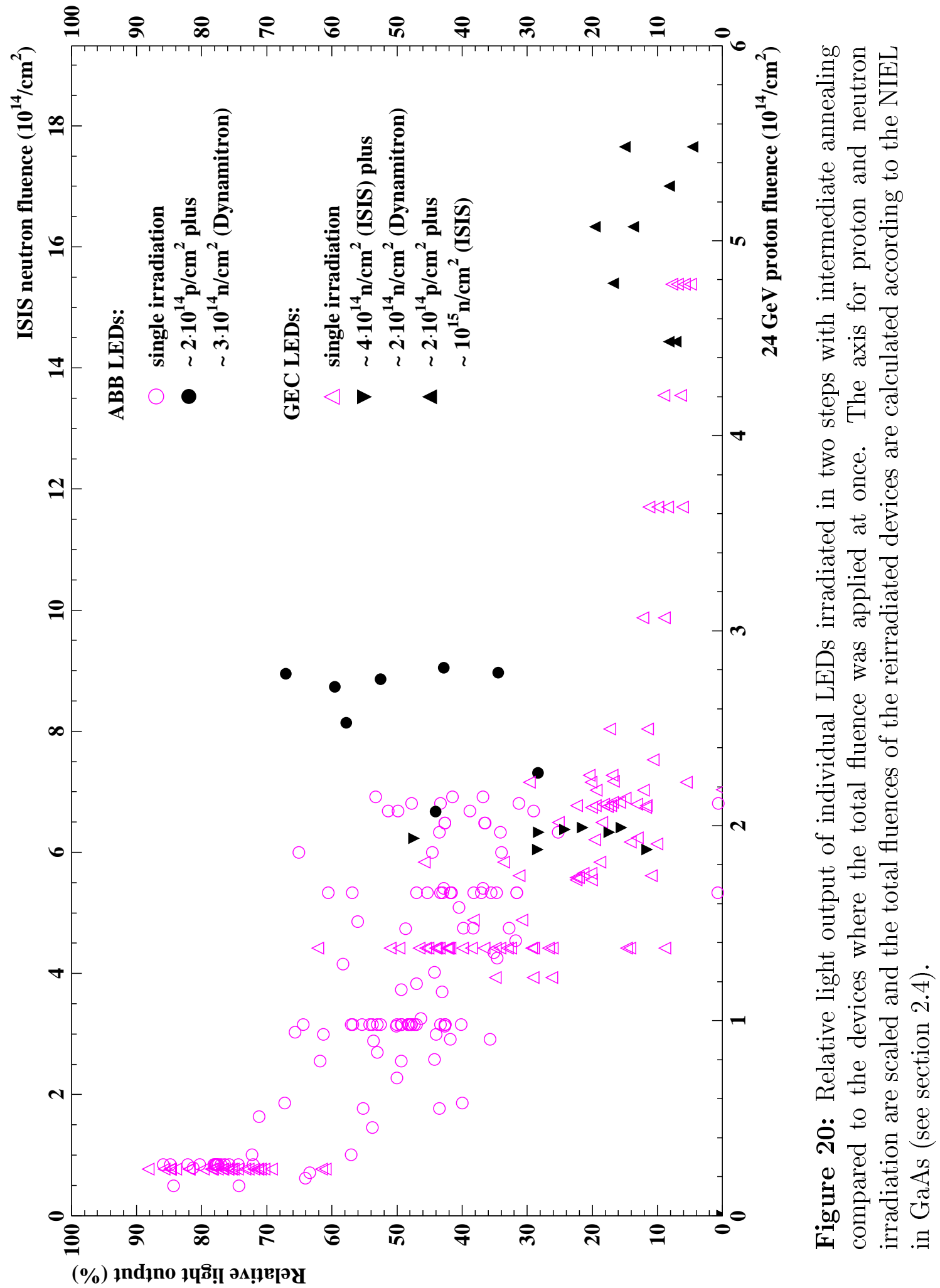




\subsection{Lifetime Studies}

\subsubsection{Acceleration of Aging with Respect to LHC}

In order to make any prediction on device life time in a reasonable amount of time, the aging of the devices studied has to be accelerated.

It is generally believed that aging of LEDs takes place only under forward bias. Thus we can achieve an acceleration factor of 12 with respect to LHC by operating the devices with a DC current. This factor is based on the estimate, that a) LHC will run about 100 days per year, b) the average link occupancy is about $50 \%$, c) the LEDs are off when no data is transmitted, and d) the 0 and 1 bits are balanced.

The acceleration of the aging of VCSELs depends on the operating mode of the optical link. If we assume that a constant bias current is needed to keep the VCSEL near or above the lasing threshold when no data is transmitted, only a factor of three could be gained with respect to the SCT by continuously operating VCSELs in DC mode.

As shown in section 2.3 an additional acceleration can be achieved by increasing the operating temperature. Because the thermal activation energy $E_{a}$ is not known for neither the LEDs nor the VCSELs tested, the acceleration factor for the operation at $50^{\circ} \mathrm{C}$ cannot be calculated with precision. However, if a nominal operating temperature of $10^{\circ} \mathrm{C}$ is assumed (the operating temperature of LEDs or VCSELs in the ATLAS SCT is expected to be between about $-10^{\circ} \mathrm{C}$ and $+10^{\circ} \mathrm{C}$ ), the typical values of $E_{a}$ found in the literature result in acceleration factors from 8 to 160 for LEDs. The thermal activation energy for VCSELs is about twice as high than the one for LEDs. Therefore, as a conservative estimate, an additional acceleration factor of 10 (35) is assumed for LEDs (VCSELs) for the aging at $50^{\circ} \mathrm{C}$.

The elevated currents applied during the annealing and in a few cases during the aging lead to an additional acceleration factor according to the Arrhenius equation (see section 2.3). However, the exponent is not known for the devices used in this test and therefore no additional acceleration factor is taken into account.

\subsubsection{Long Term Stability}

The light output of most of the devices operated at $50^{\circ} \mathrm{C}$ and at their default current remained essentially stable. However, some devices exhibited a slow antiannealing of a few percent per month during the high temperature operation, and some LEDs showed instabilities of the light output. An example of an ABB LED showing both behaviors after the irradiation with $0.85 \cdot 10^{14} \mathrm{n} / \mathrm{cm}^{2}$ is presented in figure 21a. A rather strong anti-annealing is observed by one GEC LED irradiated with $4.4 \cdot 10^{14} \mathrm{n} / \mathrm{cm}^{2}$ during the aging period (figure $21 \mathrm{~b}$ ). However, the decrease of the light output would not affect the performance of the optical link.

Figures 21c and 21d show two GEC LEDs both irradiated with $0.77 \cdot 10^{14} \mathrm{n} / \mathrm{cm}^{2}$ exhibiting a sharp drop in the light output during the high temperature operation, but showing no other irregularities. Since after the drop, the light output is still sufficient for the operation of an optical link, these LEDs were not considered as failing, too. 

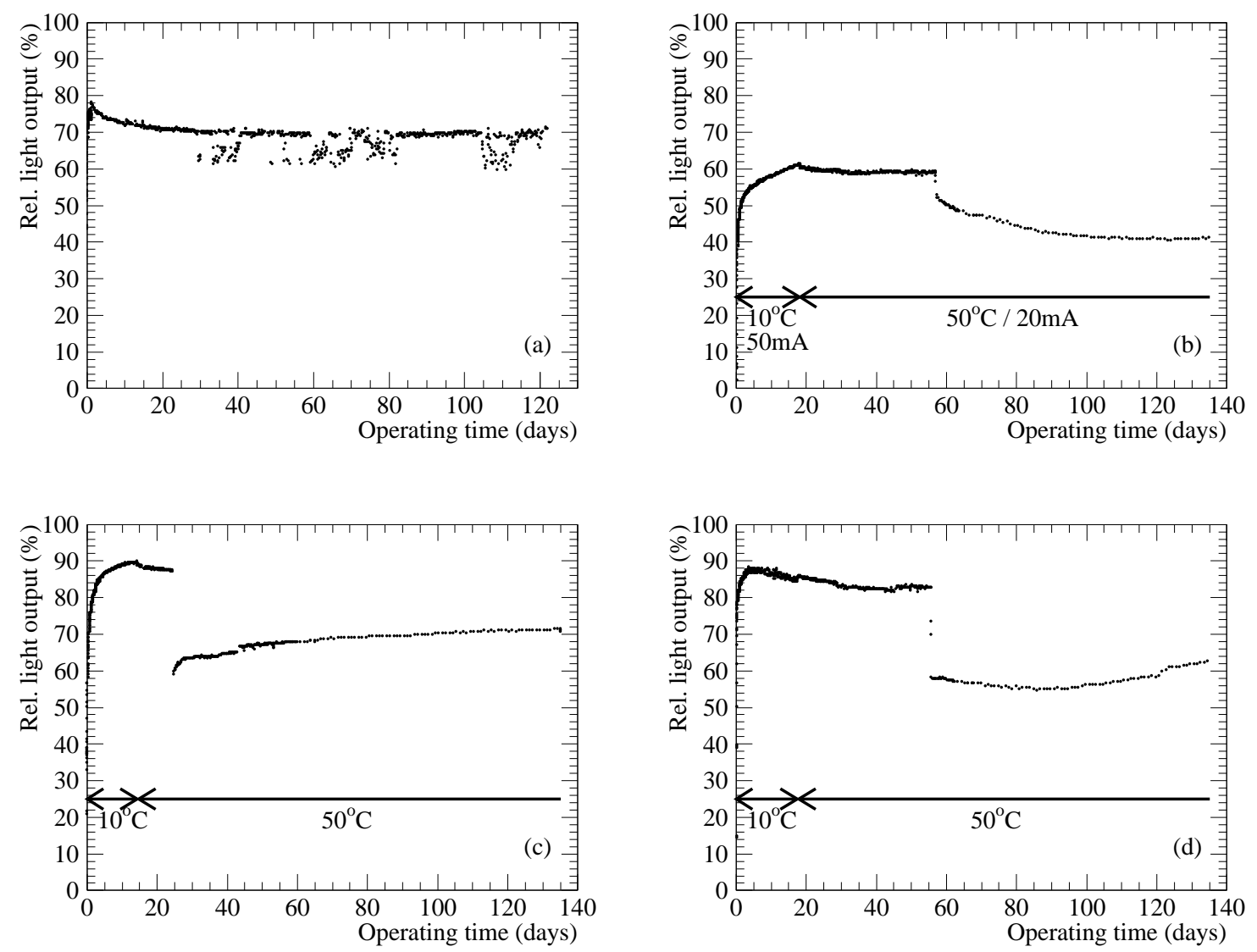

Figure 21: Long term instabilities of LEDs: a) ABB LED irradiated with $0.85 \cdot 10^{14} \mathrm{n} / \mathrm{cm}^{2}$ showing some anti-annealing and instabilities; b) a GEC LED irradiated with $4.4 \cdot 10^{14} \mathrm{n} / \mathrm{cm}^{2}$ anti-anneals during the high temperature operation; c) and d) two GEC LEDs both irradiated with $0.77 \cdot 10^{14} \mathrm{n} / \mathrm{cm}^{2}$ and operated at $20 \mathrm{~mA}$ forward current exhibiting an unexplained drop in the light output during the high temperature operation. 


\subsection{Estimated Relative Light Output after Ten Years of Operation at LHC}

The distribution of the relative light output of GEC LEDs irradiated with $24 \mathrm{GeV}$ proton fluences between $1.5 \cdot 10^{14} \mathrm{p} / \mathrm{cm}^{2}$ and $2.5 \cdot 10^{14} \mathrm{p} / \mathrm{cm}^{2}$ and annealed at forward currents between $50 \mathrm{~mA}$ and $80 \mathrm{~mA}$ is shown in figure 22. The left histogram shows the relative light output distribution after the annealing, and the right histogram shows the corresponding distribution after operating the LEDs at $50^{\circ} \mathrm{C}$ for up to about 7 months, corresponding to up to about 70 years of operation in the ATLAS SCT. As can be seen, during the high temperature operation the relative light output remained essentially stable.

Although the LEDs included into the figure were irradiated with fluences up to about twice as high as those currently estimated for the ATLAS SCT during 10 years of operation, the relative light output distribution represents a reasonable approximation to the relative light output distribution, given the large uncertainties in the fluence calculation, that can be expected for GEC LEDs after 10 years of operation at LHC.
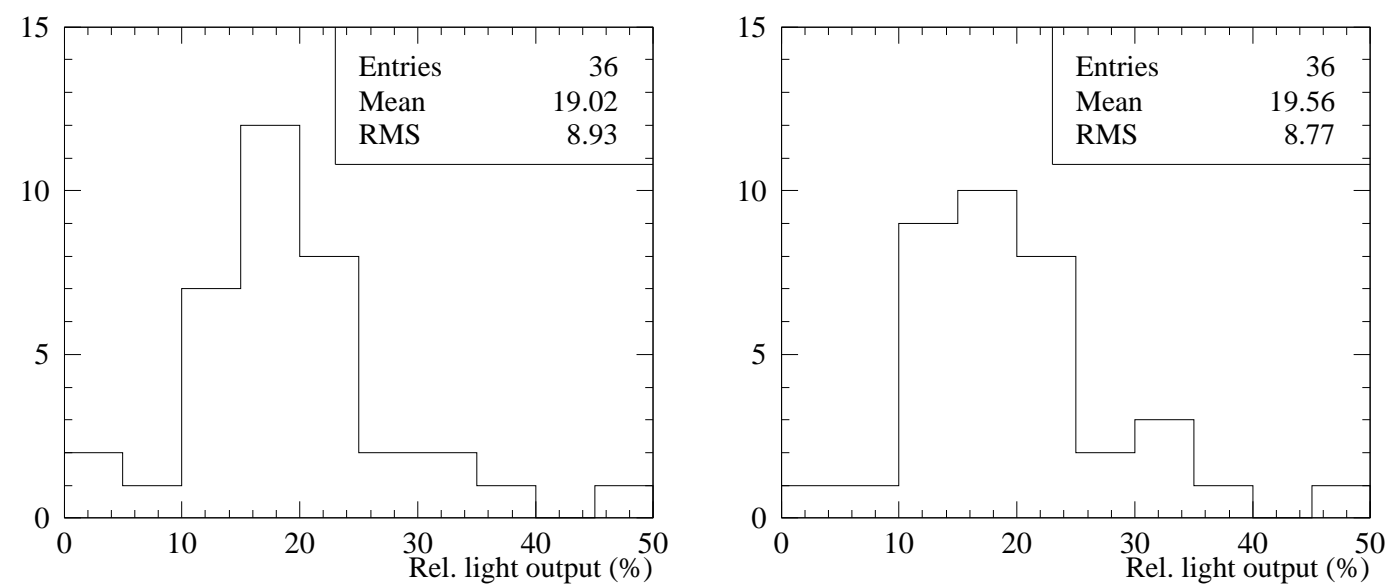

Figure 22: Distribution of relative light output of GEC LEDs irradiated with fluences between $1.5 \cdot 10^{14} \mathrm{p} / \mathrm{cm}^{2}$ and $2.5 \cdot 10^{14} \mathrm{p} / \mathrm{cm}^{2}$ after annealing at maximum forward currents between $50 \mathrm{~mA}$ and $80 \mathrm{~mA}$ (left) and after the high temperature operation (right).

\subsection{Statistics of Irradiated Devices}

Table 8 shows the operating time achieved and the estimated equivalent operating time in the SCT for the different devices. All devices used for the statistics have been irradiated with a minimum fluence of $2 \cdot 10^{14} \mathrm{n} / \mathrm{cm}^{2}$ or $0.6 \cdot 10^{14} \mathrm{p} / \mathrm{cm}^{2}$, where the proton fluence corresponds to the neutron fluence if the radiation damage scales according to the NIEL hypothesis (see section 2.4). 


\begin{tabular}{|c|c|c|c|c|c|c|c|c|c|c|c|c|c|c|c|c|}
\hline 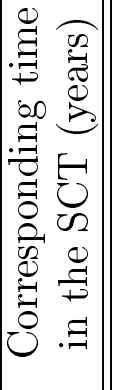 & i & $\dot{\stackrel{\Omega}{े}}$ & 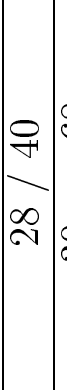 & $\begin{array}{c}\infty \\
\vdots \\
\vdots \\
0 \\
0\end{array}$ & -5 & 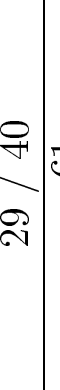 & \begin{tabular}{c|c}
-1 & 0 \\
2 & 2
\end{tabular} & 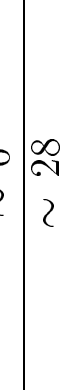 & $\left|\begin{array}{l}0 \\
2\end{array}\right|$ & $\mid \begin{array}{c}F \\
\vdots \\
-\end{array}$ & \begin{tabular}{l|l}
0 & $F$ \\
2 & 2
\end{tabular} & $\begin{array}{ll}0 \\
2 \\
2\end{array}$ & & \begin{tabular}{l|l}
0 & $\exists$ \\
2 & $z$ \\
2
\end{tabular} & $\begin{array}{l}0 \\
2\end{array}$ & 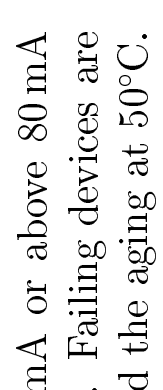 \\
\hline 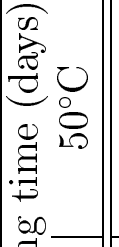 & 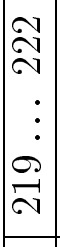 & $\begin{array}{c}\stackrel{N}{N} \\
\vdots \\
\vdots \\
2 \\
20\end{array}$ & $\begin{array}{l}-\vec{N} \\
- \\
-1 \\
\infty \\
\infty\end{array}$ & 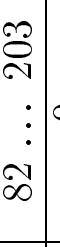 & 0 & 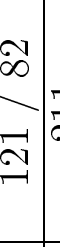 & $\underset{\sim}{\vec{N}}$ & $\%$ & 0 & 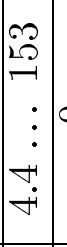 & $0 \stackrel{2}{0}$ & $\begin{array}{c}0 \\
0 \\
t\end{array}$ & 80 & 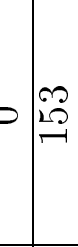 & 0 & 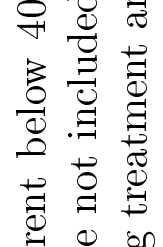 \\
\hline 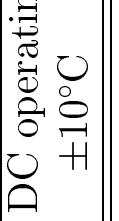 & $\begin{array}{c}20 \\
20 \\
\vdots \\
-1\end{array}$ & $\begin{array}{c}\vec{F} \\
\vdots \\
0\end{array}$ & $\begin{array}{c}\overrightarrow{1} \\
\vdots \\
\vdots \\
10 \\
-1\end{array}$ & $\begin{array}{r}尺 \\
\vdots \\
\infty \\
\infty \\
\hdashline\end{array}$ & $\underset{1}{q}$ & $\begin{array}{c}\infty \\
\infty \\
\infty \\
\infty\end{array}$ & & 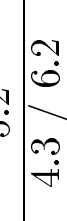 & $\mid \begin{array}{c}0 \\
2 \dot{0} \\
0 .\end{array}$ & 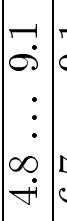 & 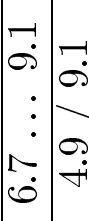 & 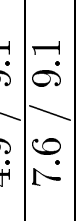 & 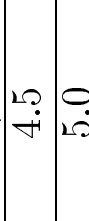 & $\dot{D}_{\dot{0}} \underset{2 \dot{0}}{0}$ & $\vec{\leftrightarrow}$ & 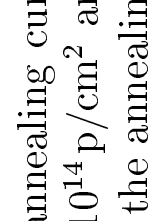 \\
\hline 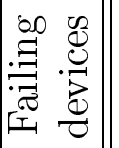 & -1 & N & 0 & -1 & -10 & $\infty$ & 0 & 0 & 0 & -10 & 1010 & 0 & 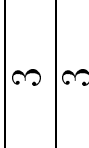 & $r 0$ & 0 & $\begin{array}{cc}0 & 0 \\
\frac{\pi}{7} & \overline{0} \\
\overrightarrow{3} & 0\end{array}$ \\
\hline 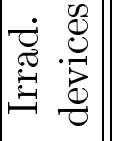 & 今. & î & $\mathscr{D}$ & $\begin{array}{ll}\infty & \\
\infty & 0\end{array}$ & $\infty$ & $\stackrel{\sim}{\sim} 0$ & $\infty \mid \infty$ & 0 & $\underset{c \mid}{0}$ & 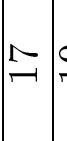 & $\because 2$ & \begin{tabular}{ll|}
2 & 20 \\
-1
\end{tabular} & $\infty$ & 010 & $N$ & 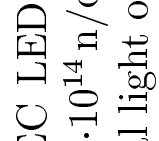 \\
\hline 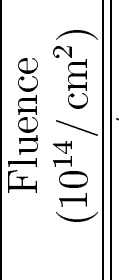 & 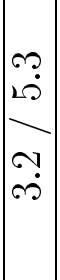 & $\begin{array}{c}\mathcal{N} \\
\stackrel{v}{ } \\
\vdots \\
0 \\
\dot{0}\end{array}$ & $\underset{\forall}{\nabla}$ & 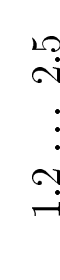 & $\begin{array}{l}? \\
\vdots \\
\vdots \\
\vdots \\
\end{array}$ & $\begin{array}{c}\infty \\
+ \\
+ \\
\vdots \\
0 \\
\dot{+} \\
\dot{0}\end{array}$ & $\begin{array}{c}\overrightarrow{\text { ij }} \\
2\end{array}$ & & 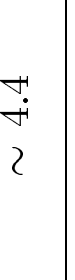 & $\stackrel{\infty}{-}$ & - & $\begin{array}{r}\text { r } \\
2\end{array}$ & $\begin{array}{r}\overrightarrow{+} \\
\dot{+} \\
2\end{array}$ & & $\begin{array}{l}r \\
2 \\
2\end{array}$ & 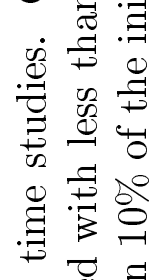 \\
\hline 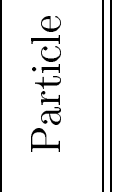 & $=$ & 2 & $=$ & & 2 & & 2 & & $\approx$ & & $\rho$ & & 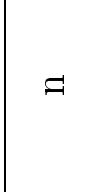 & & 2 & 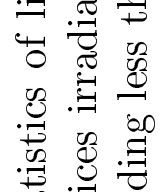 \\
\hline$\frac{\mathscr{e}}{\stackrel{0}{3}}$ & $\begin{array}{l}\stackrel{5}{\mid c} \\
\frac{9}{6}\end{array}$ & & & $\begin{array}{l}\text { 空 } \\
\text { 足 } \\
\text { 至 }\end{array}$ & & & 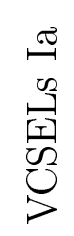 & & & 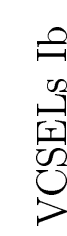 & 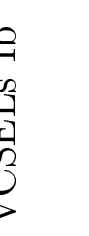 & & & 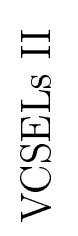 & & 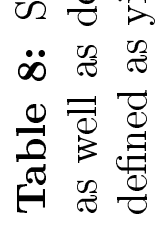 \\
\hline
\end{tabular}


Devices with a light output less than $10 \%$ after the annealing treatment and after the aging are considered as dead. This number is much lower than the $50 \%$ used as the standard definition in industry and has been derived from the $\mathrm{S} / \mathrm{N}$ requirement of the optical link proposed for the SCT.

As mentioned in section 3.2.1, LEDs with a light output lower than $5 \mu \mathrm{W}$ coupled into a multimode fibre were not included into the statistics in order to avoid any bias from very weak LEDs. In order to use the same criteria, VCSELs yielding less than $10 \%$ of the initial light output are considered as dead, too. However, a VCSEL with $10 \%$ of the initial light output will still yield more light than a bright LED before the irradiation.

As shown in section 5.3.2, the annealing current for the GEC LEDs must be in the range of $40-80 \mathrm{~mA}$ in order to have a considerable annealing after the irradiation. Therefore, GEC LEDs with a maximum annealing current below or above this range are excluded from the statistics.

3 out of 91 ABB LEDs and 2 out of 65 GEC LEDs with a maximum fluence of $2.5 \cdot 10^{14} \mathrm{p} / \mathrm{cm}^{2}$ or $5.5 \cdot 10^{14} \mathrm{n} / \mathrm{cm}^{2}$ failed right after the irradiation or during the annealing. After the irradiation with even higher fluences, the GEC LEDs yielded almost no light and showed only a very slow annealing at $50 \mathrm{~mA}$. Only one out of 12 devices has reached a relative light output above $10 \%$ after an annealing period of up to 70 days. However, after another $80-120$ days of operation at $50^{\circ} \mathrm{C}$ and at forward currents of $50-80 \mathrm{~mA}$, another 3 devices yield more than $10 \%$ relative light output.

The VCSELs of type Ia and Ib seem to be very radiation hard. Only 1 out of 113 devices irradiated with fluences up to $3.7 \cdot 10^{14} \mathrm{p} / \mathrm{cm}^{2}$ failed. However, the VCSELs of type II have shown 6 failures in a total of 21 devices irradiated with $3.7 \cdot 10^{14} \mathrm{p} / \mathrm{cm}^{2}$ or $4.4 \cdot 10^{14} \mathrm{n} / \mathrm{cm}^{2}$. Although the statistics is low, it seems that this type of VCSEL is less radiation hard than the devices with a higher threshold current.

No devices failed during the long term operation at $50{ }^{\circ} \mathrm{C}$, but some antiannealing and unexplained drops in the relative light output were observed for a few LEDs (see section 5.6.2).

\subsection{Behavior of Failing Devices}

In this section the failure characteristics of the LEDs and VCSELs are summarized. As in the previous section, only devices irradiated with at least $2 \cdot 10^{14} \mathrm{n} / \mathrm{cm}^{2}$ or $0.6 \cdot 10^{14} \mathrm{p} / \mathrm{cm}^{2}$ and GEC LEDs annealed at a forward current between 40 and $80 \mathrm{~mA}$ are considered. An overview of the failing devices irradiated with less than $6 \cdot 10^{14} \mathrm{n} / \mathrm{cm}^{2}$ or $2.5 \cdot 10^{14} \mathrm{p} / \mathrm{cm}^{2}$ is given in table 9 .

The following categories of failures were found:

- The LED behaves no longer as a diode but as an ohmic resistor. This is the case for one ABB LED after $0.7 \cdot 10^{14} \mathrm{p} / \mathrm{cm}^{2}$ and one GEC LED after $2.2 \cdot 10^{14} \mathrm{p} / \mathrm{cm}^{2}$ (figure 23a). The latter one gave some light after the irradiation, but died within about $30 \mathrm{~min}$ of operation as shown in figure $23 \mathrm{~b}$. 


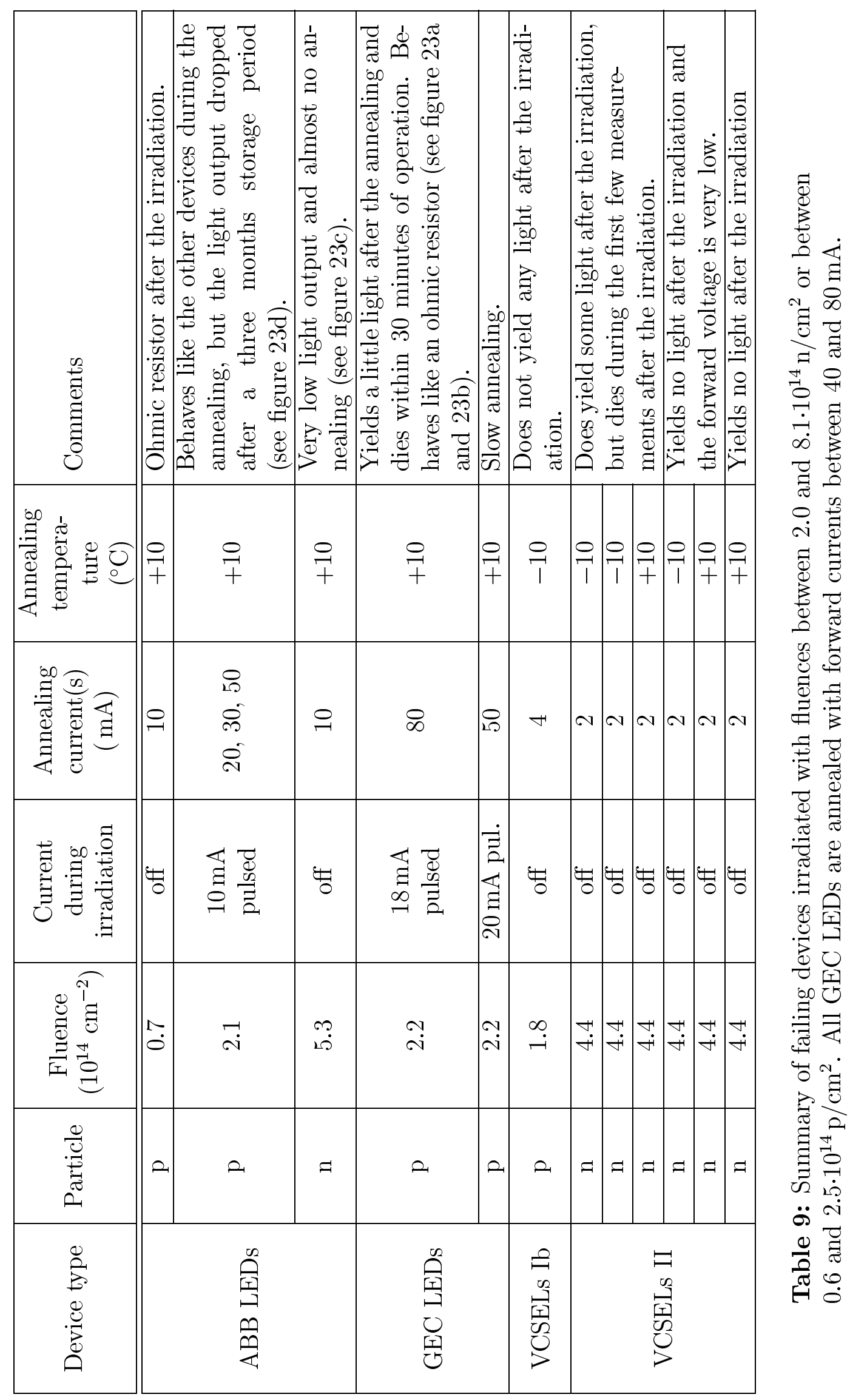



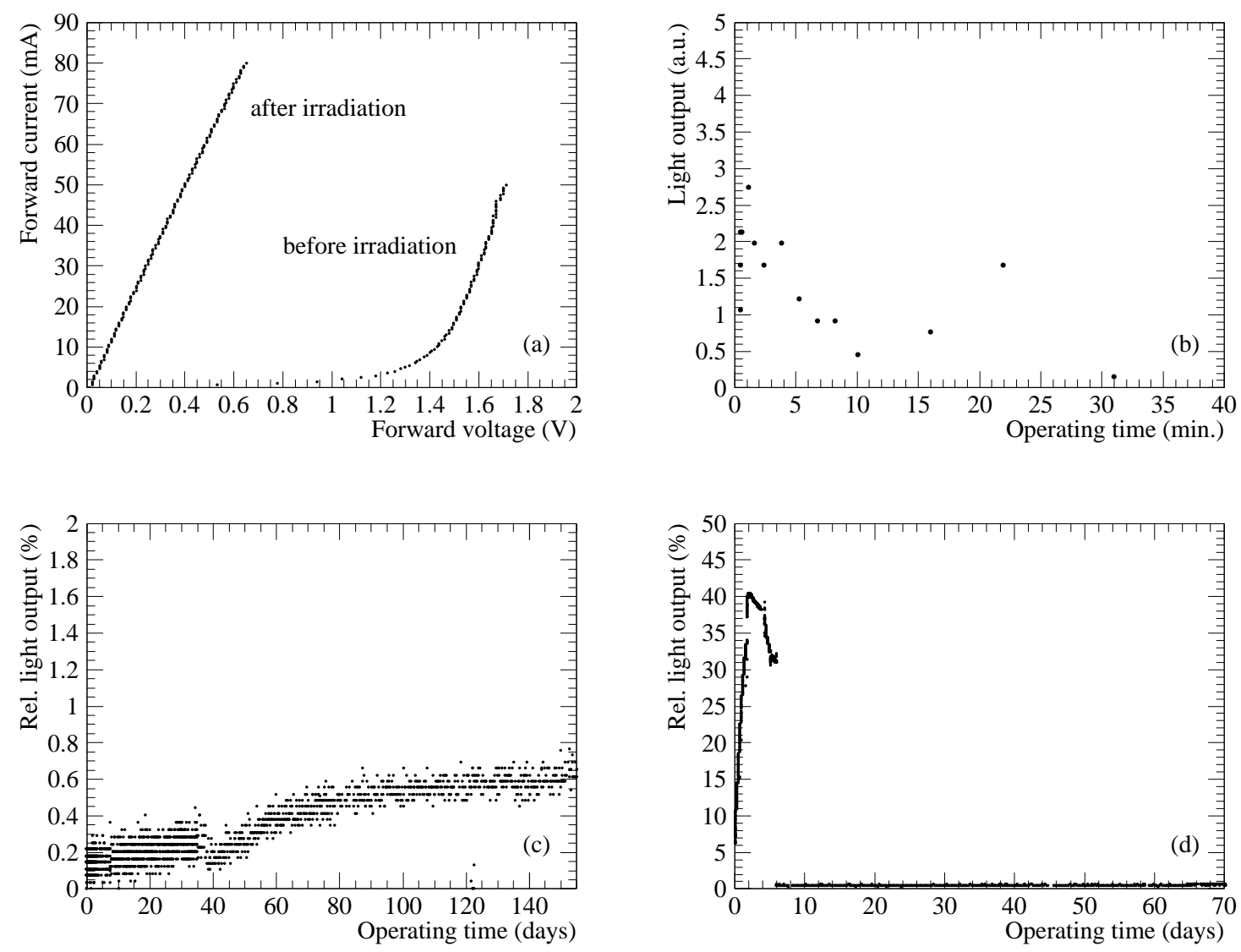

Figure 23: Some examples of failing LEDs: a) this GEC LED behaves after the irradiation with $2.2 \cdot 10^{14} \mathrm{p} / \mathrm{cm}^{2}$ no longer as a diode but as an ohmic resistor; b) a GEC LED dying within the first minutes of annealing after irradiation with $2.2 \cdot 10^{14} \mathrm{p} / \mathrm{cm}^{2}$; c) no significant annealing is seen for this ABB LED after $5.3 \cdot 10^{14} \mathrm{n} / \mathrm{cm}^{2}$ under a forward current of $10 \mathrm{~mA}$; d) another ABB LED showed annealing and anti-annealing after the irradiation with $2.1 \cdot 10^{14} \mathrm{p} / \mathrm{cm}^{2}$, but yielded almost no light after a storage period of three months at room temperature. 
- The light output of the device is very low and no significant annealing at a given forward current was observed, in contrast to the other devices on the same module. One ABB LED after $5.3 \cdot 10^{14} \mathrm{n} / \mathrm{cm}^{2}$ under a forward current of $10 \mathrm{~mA}$ (figure $23 \mathrm{c}$ ) and one GEC LED after $2.2 \cdot 10^{14} \mathrm{p} / \mathrm{cm}^{2}$ under a forward current of $50 \mathrm{~mA}$ fall into this category.

- The LED anneals after the irradiation, but after a storage period of three months at room temperature, the light output was decreased to a few percent. This behavior has been observed for one ABB LED after $2.1 \cdot 10^{14} \mathrm{p} / \mathrm{cm}^{2}$ (figure 23d).

- The VCSEL does not yield any light after the irradiation, but still shows the normal diode characteristics. This is the case for one VCSEL Ib after the irradiation with $1.8 \cdot 10^{14} \mathrm{p} / \mathrm{cm}^{2}$ and one VCSEL II after the irradiation with $4.4 \cdot 10^{14} \mathrm{n} / \mathrm{cm}^{2}$.

- The light output after the irradiation is comparable to the other devices irradiated with the same fluence, but the VCSEL died within seconds when operated. This behavior was seen by three VCSELs of type II after the irradiation with $4.4 \cdot 10^{14} \mathrm{n} / \mathrm{cm}^{2}$.

- There is no light output after the irradiation and the forward voltage is very low. This is the case for two VCSELs II after the irradiation with $4.4 \cdot 10^{14} \mathrm{n} / \mathrm{cm}^{2}$.

\section{Conclusions}

The short term annealing behavior and the life time of a large number of LEDs and VCSELs were measured after irradiation with neutron and proton fluences beyond those expected at the inner tracker of ATLAS. The VCSELs produced by Sandia National Laboratories were found to be much more radiation hard than both kind of LEDs tested in this study which were manufactured by GEC-Marconi and ABB Hafo, respectively. Moreover, at an operation current of only $4 \mathrm{~mA}$ the light output of these low threshold VCSELs is nearly two orders of magnitude larger as for LEDs with a 3 to 5 times higher operating current.

The measured reduction of the light output of the GEC LEDs after irradiation was used to determine the damage constants both for $24 \mathrm{GeV}$ protons and for the $\sim 1 \mathrm{MeV}$ spallation neutrons at the RAL ISIS facility. The resulting proton to neutron damage ratio of $3.2 \pm 0.1$ agrees perfectly with the ratio obtained from calculations of the non-ionizing energy loss (NIEL) in GaAs. This result supports strongly the NIEL hypothesis on the degradation of LEDs by irradiation, which is crucial for the extrapolation of these radiation hardness studies to the LHC environment. If scaling of the radiation damage in GaAs according to the NIEL hypothesis is assumed, the currently estimated total fluence for the innermost barrel layer in the ATLAS SCT corresponds to a $24 \mathrm{GeV}$ proton fluence of about $1.5 \cdot 10^{14} \mathrm{p} / \mathrm{cm}^{2}$, with an uncertainty of roughly $50 \%$. 
The light yield of the GEC LEDs, which were irradiated with about $2 \cdot 10^{14} \mathrm{p} / \mathrm{cm}^{2}$, was typically reduced by a factor of 50 to 200, and about a factor of 30 for the ABB LEDs after the same proton fluence. However, the radiation damage could be partially annealed by driving the LEDs at a forward current of 40 to $80 \mathrm{~mA}$ continuously for a few days to several weeks, resulting in a relative light output with respect to the one before the irradiation of $10 \%$ to $20 \%$ for the GEC LEDs and of $20 \%$ to $40 \%$ for the ABB LEDs. Unfortunately the ABB LEDs are no more available, because the company was sold.

The VCSELs, which were pulsed with the normal operating current during the irradiation, showed essentially no degradation up to fluences of about $4 \cdot 10^{14} \mathrm{p} / \mathrm{cm}^{2}$, whereas for those which were off during the irradiation the lasing threshold current increased. Complete annealing of this radiation damage was achieved by driving the VCSELs for at most a few days at the normal operating current.

A few LEDs died during or soon after the irradiation with fluences in the range of $2 \cdot 10^{14} \mathrm{n} / \mathrm{cm}^{2}$ to $8 \cdot 10^{14} \mathrm{n} / \mathrm{cm}^{2}$ or $0.6 \cdot 10^{14} \mathrm{p} / \mathrm{cm}^{2}$ to $2.5 \cdot 10^{14} \mathrm{p} / \mathrm{cm}^{2}$, namely 2 out of 65 GEC LEDs and 3 out of 91 ABB Hafo LEDs. Also 1 of the 113 VCSELs with a threshold current of $\sim 2 \mathrm{~mA}$, which were irradiated either with about $4 \cdot 10^{14} \mathrm{n} / \mathrm{cm}^{2}$ or with about $2 \cdot 10^{14} \mathrm{p} / \mathrm{cm}^{2}$ to $4 \cdot 10^{14} \mathrm{p} / \mathrm{cm}^{2}$, was found dead after the irradiation. The 21 VCSELs with a threshold current below $1 \mathrm{~mA}$ turned out to be much less robust: 6 of them died after irradiation with about $4 \cdot 10^{14} \mathrm{n} / \mathrm{cm}^{2}$. All the LEDs and VCSELs, which survived the irradiation and the annealing treatment showed stable performance during the subsequent long term test at $50^{\circ} \mathrm{C}$, which is equivalent to up to 70 years of operation at LHC.

This study showed clearly, that the tested VCSELs with a threshold current of about $2 \mathrm{~mA}$ would be the best choice for the optical readout of the SCT (and eventually of the pixel layers). The problem of the packaging, however, is more difficult for VCSELs than for LEDs, because reflections can cause huge noise. At the time of this writing it is not sure if radiation hard and affordable VCSELs-tofibre couplings can be developed.

For the GEC LEDs the packaging problem is already solved. According to the ATLAS Technical Design Report [1], the anticipated reduced light output after annealing of these devices still allows for safe operation of digital optical links at $40 \mathrm{Mb} / \mathrm{s}$, but requires PIN-diode receivers with excellent noise performance. LEDs with an initial light output of less than about $15 \mu \mathrm{W}$ should be used only at the outermost layers of the SCT or even be rejected. A special annealing procedure is needed during the shut down periods of the LHC, during which the LEDs must be driven at a current of about $50 \mathrm{~mA}$ for several days to several weeks.

The observed failure rates of $\sim 1 \%$ for the VCSELs with $\sim 2 \mathrm{~mA}$ threshold and $\sim 3 \%$ for the GEC LEDs are rather high, but are tolerable in view of the foreseen redundancy scheme for the SCT readout. 


\section{Acknowledgments}

We are indebted to the technical staff of the Bern group for the construction and maintenance of the scanning machine and its electronics. We thank John H. Bibby for mounting part of the LEDs onto the ceramic boards used in the scanning machine, and we greatly appreciate the support of Mike Edwards, Maurice Glaser, François Lemeilleur, Steve Oglesby and their teams who performed the irradiation of the LEDs and VCSELs. Finally, we acknowledge the support of the Swiss National Foundation and the U.K. Particle Physics and Astronomy Research Council.

\section{References}

[1] ATLAS Collaboration, ATLAS Inner Detector Technical Design Report, Volume 2, ATLAS TDR 5, CERN/LHCC/97-17 (1997)

[2] PIN Diode Irradiation, University of Birmingham, ATLAS Internal Note in preparation

[3] J. Beringer, Studies for the Inner Detector of the ATLAS Experiment at the Large Hadron Collider, PhD Thesis, University of Bern, 1996

[4] R.K. Mommsen, Life Time Studies of Irradiated LEDs and VCSELs for the ATLAS Experiment at the Large Hadron Collider, diploma thesis, University of Bern, 1997

[5] J. Beringer et al., A life time test of neutron irradiated light emitting diodes, Nucl. Instr. and Meth. A373 (1996) 320

[6] I. Dawson et al., Irradiation tests of optoelectronic components for LHC innerdetectors, Nucl. Instr. and Meth. A387 (1997) 369

[7] ATLAS Collaboration, ATLAS Liquid Argon Calorimeter Technical Design Report, ATLAS TDR 3, CERN/LHCC/96-41 (1996)

[8] K. Gill et al., Effect of neutron irradiation of $M Q W$ lasers to $10^{15} \mathrm{n} / \mathrm{cm}^{2}$, CMS Note 1997/044

[9] J. Beringer et al., First Results of a Comprehensive Life Time Test of Irradiated LEDs and VCSELs, Proceedings of the 2nd Workshop of Electronics for LHC Experiments, Balatonfüred, Hungary, 1996, CERN/LHCC/96-39

[10] M.H. Pilkuhn, Light Emitting Diodes, in ed. T.S. Moss, Handbook on Semiconductors, volume 4, North-Holland (1981)

[11] Hewlett Packard, Reliability Considerations in Designing Fiber Optic Transmitters, Optocoupler and Fiber Optics Applications Handbook (1986)

[12] K. Gillessen and W. Schairer, Light Emitting Diodes, Prentice-Hall International (UK) Ltd. (1987) 
[13] N.K. Dutta and C.L. Zipfel, Reliability of Lasers and LEDs, in eds. S.E. Miller and I.P. Kaminow, Optical Fibre Telecommunications II, Academic Press Inc. (1988)

[14] M. Fukuda, Reliability and Degradation of Semiconductor Lasers and LEDs, Artech House Inc. (1991)

[15] Honeywell, Long-Term Power Output Reliability of Cap Rock (SEC589) LED Chip, Optoelectronics Fiber Optic Products, Catalog E27 (1993)

[16] C.-C. Wu et al., Reliability of Proton Implanted Vertical Cavity Surface Emitting Lasers, Intern. J. of High Speed Electr. and Systems, vol 5, no. 4, p. 731-764 (1994)

[17] P.M. Petroff, Physics and materials issues behind the lifetime problem in semiconductor lasers and light emitting diodes, Proceedings of the SPIE, vol. 2683, pp. 52-58, SPIE, San Jose, CA (1996)

[18] SCT group, ATLAS SCT Technical Proposal Backup Document, ATLAS Internal Note, INDET-NO-085 (1995)

[19] J.K. Guenter et al., Reliability of proton-implanted VCSELs for data communications, Proceedings of the SPIE, vol. 2683, pp. 102-113, SPIE, San Jose, CA (1996)

[20] K.L. Lear et al., Life-testing oxide confined VCSELs: too good to last?, Proceedings of the SPIE, vol. 2683, pp. 114-122, SPIE, San Jose, CA (1996)

[21] B. Twu and H. Kung, Reliability of fiber optic emitters, in ed. D. Hall, Integrated Optics II, SPIE vol. 321 (1982)

[22] D.V. Lang and L.C. Kimerling, Observation of Recombination-Enhanced Defect Reactions in Semiconductors, Phys. Rev. Lett. 33 (1974) 489

[23] P.W. Hutchinson et al., Defect structure of degraded heterojunction GaAlAsGaAs lasers, Appl. Phys. Lett., vol. 26, no. 5 (1975) 250

[24] P. Petroff and R.L. Hartman, Defect structure introduced during operation of heterojunction GaAs lasers, J. Appl. Phys. 23(8) (1973) 469

[25] P. Petroff and R.L. Hartman, Rapid degradation phenomenon in heterojunction GaAlAs-GaAs lasers, J. Appl. Phys. 45(9) (1974) 3899

[26] P.M. Petroff, O.G. Lorimor and J.M. Ralston, Defect structure induced during forward-bias degradation of GaP green-light-emitting diodes, J. Appl. Phys. 74(4) (1976) 1583

[27] L.C. Kimerling, P. Petroff and H.J. Leamy, Injection-stimulated dislocation motion in semiconductors, Appl. Phys. Lett., vol. 28, no. 6 (1976) 297 
[28] O. Ueda et al., Defect structure of degraded $G a_{1-x} A l_{x} A s$ double-heterostructure light-emitting diodes, J. Appl. Phys. 50(2) (1979) 765

[29] S. Kishino et al., Dark-line defects induced by mechanical bending in GaAs$G a_{1-x} A l_{x} A s$ double-heterostructure wafers, Appl. Phys. Lett., vol. 29, no. 8 (1976) 488

[30] G. Zaeschmar and R.S. Speer, Mechanical-stress-induced degradation in homojunction GaAs LEDs, J. Appl. Phys. 50(9) (1979) 5686

[31] R. Ramakumar, Engineering Reliability: Fundamentals and Applications, Prentice-Hall Int., Inc., Englewood Cliffs, New Jersey, USA, 1993

[32] J. Lindhard et al., Range concepts and heavy ion ranges, Kgl. Dan. Vidensk. Selsk. Mat.-Fys. Medd., vol. 33 (1963) 3

[33] J. Lindhard et al., Integral Equations Governing Radiation Effects, Kgl. Dan. Vidensk. Selsk. Mat.-Fys. Medd., vol. 33, no. 10 (1963) 2

[34] V.A.J. van Lint, R.E. Leadon and J.F. Colwell, Energy Dependence of Displacement Effects in Semiconductors, IEEE Trans. Nucl. Sci., vol. NS-19, no. 6 (1972) 181

[35] S. Wood et al., Simulation of Radiation Damage in Solids, IEEE Trans. Nucl. Sci., vol. NS-28, no. 6 (1981) 4107

[36] G.P. Mueller, N.D. Wilsey and M. Rosen, The Structure of Displacement Cascades in Silicon, IEEE Trans. Nucl. Sci., vol. NS-29, no. 6 (1982) 1493

[37] L.W. Aukerman et al., Radiation Effects in GaAs, J. Appl. Phys. 34(12) (1963) 3590

[38] C.J. Dale et al., High Energy Electron Induced Displacement Damage in Silicon, IEEE Trans. Nucl. Sci., vol. NS-35, no. 6 (1988) 1208

[39] G.P. Summers et al., Damage Correlations in Semiconductors Exposed to Gamma, Electron and Proton Radiations, IEEE Trans. Nucl. Sci., vol. NS-40, no. 6 (1993) 1372

[40] A.L. Barry et al., The Energy Dependence of Lifetime Damage Constants in GaAs LEDs for 1-500 MeV Protons, IEEE Trans. Nucl. Sci., vol. NS-42, no. 6 (1993) 2104

[41] T.F. Luera et al., Neutron Damage Equivalence for Silicon, Silicon Dioxide, and Gallium Arsenide, IEEE Trans. Nucl. Sci., vol. NS-34, no. 6 (1987) 1557

[42] G.P. Summers et al., Correlation of Particle-Induced Displacement Damage in Silicon, IEEE Trans. Nucl. Sci., vol. NS-34, no. 6 (1987) 1134 
[43] G.P. Summers et al., Displacement Damage in GaAs Structures, IEEE Trans. Nucl. Sci., vol. NS-35, no. 6 (1988) 1221

[44] A. van Ginneken, Non Ionizing Energy Deposition in Silicon for Radiation Damage Studies, FERMILAB report FN-522 (1989)

[45] A.M. Ougouag et al., Differential Displacement Kerma Cross Section for Neutron Interactions in Si and GaAs, IEEE Trans. Nucl. Sci., vol. NS-37, no. 6 (1990) 2219

[46] M. Huhtinen and P.A. Aarnio, Pion induced displacement damage in silicon devices, Nucl. Instr. and Meth. A335 (1993) 580

[47] A. Chilingarov, J.S. Meyer and T. Sloan, Radiation Damage due to NIEL in GaAs Particle Detectors, ATLAS Internal Note, INDET-NO-134 (1996)

[48] P.A. Aarnio et al., Damage observed in silicon diodes after low energy pion irradiation, Nucl. Instr. and Meth. A360 (1995) 521

[49] S. Oglesby, private communication

[50] B.H. Rose and C.E. Barnes, Proton damage effects on light emitting diodes, J. Appl. Phys. 53(3) (1982) 1772

[51] C.E. Barnes, Radiation effects on light sources and detectors, SPIE Vol. 541 Radiation Effects in Optical Materials (1985), p.138

[52] G.C. Messenger, A Summary Review of Displacement Damage from High Energy Radiation in Silicon Semiconductors and Semiconductor Devices, IEEE Trans. Nucl. Sci., vol. NS-39, no. 3 (1992) 468

[53] C.E. Barnes, Effects of $\mathrm{Co}^{60}$ Gamma Irradiation on Epitaxial GaAs Laser Diodes, Phys. Rev. B1 (1970) 4735

[54] A.L. Barry, R. Wojcik and A.L. MacDiarmid, Response of GaAs Displacement Damage Monitors to Protons, Electrons, and Gamma Irradiation, IEEE Trans. Nucl. Sci., vol. NS-36, no. 6 (1989) 2400

[55] A.L. Barry et al., An Improved Displacement Damage Monitor, IEEE Trans. Nucl. Sci., vol. NS-37, no. 6 (1990) 1726

[56] B. Dinkespiler et al., Neutron Irradiation of Optical Link Components, ATLAS Internal Note, LARG-NO-002 (1994)

[57] D. Bräunig et al., Radiation-Induced Displacement Damage in Silicon Carbide Blue Light-Emitting Diodes, IEEE Trans. Nucl. Sci., vol. NS-39, no. 3 (1992) 428

[58] D.V. Lang, Deep-level transient spectroscopy: A new method to characterize traps in semiconductors, J. Appl. Phys. 45(7) (1974) 3023 
[59] B. Dinkespiler et al., Analogue Optical Links for the Front-End Read Out of the ATLAS Liquid Argon Calorimeter, Proceedings of the 2nd Workshop of Electronics for LHC Experiments, Balatonfüred, Hungary, 1996, CERN/LHCC/96-39

[60] K.D. Choquette et al., Fabrication and Performance of Selectively Oxidized Vertical-Cavity Lasers, IEEE Photonics Techn. Letters, vol. 7, no. 11 (1995)

[61] M. Osiński and W. Nakwaski, Thermal Effects in Vertical-Cavity SurfaceEmitting Lasers, Intern. J. of High Speed Electr. and Systems, vol 5, no. 4, p. $667-730$ (1994)

[62] Y.A. Akulova et al., Low-Temperature Optimized Vertical-Cavity Lasers with Submilliamp Threshold Currents over the 7r-370K Temperature Range, IEEE Photonics Techn. Letters, vol. 9, no. 3 (1997)

[63] S. Jiang, M. Dagenais and R.A. Morgan, Spectral Characteristics of VerticalCavity Surface-Emitting Lasers with Strong External Optical Feedback, IEEE Photonics Techn. Letters, vol. 7, no. 7 (1995)

[64] D.M. Kuchta et al., Relative intensity noise of vertical cavity surface emitting lasers, Appl. Phys. Lett., vol. 62, no. 11 (1993) 1194

[65] T.E. Sale, Vertical Cavity Surface Emitting Lasers, John Wiley \& Sons Inc., New York 1995

[66] C.J. Chang-Hasnain et al., Dynamic, Polarization, and Transverse Mode Characteristics of Vertical Cavity Surface Emitting Lasers, IEEE J. of Quantum Electronics, vol. 27, no. 6 (1991)

[67] D. Vakhshoori et al., Top-surface emitting lasers with $1.9 \mathrm{~V}$ threshold voltage and the effect of spatial hole burning on their transverse mode operation and efficiencies, Appl. Phys. Lett., vol. 62, no. 13 (1993) 1448

[68] G.C. Wilson et al., Spatial hole burning and self-focusing in vertical-cavity surface-emitting laser diodes, Appl. Phys. Lett., vol. 64, no. 5 (1994) 542

[69] A. Valle, J. Sarma and K.A. Shore, Spatial Holeburning Effects on the Dynamics of Vertical Cavity Surface-Emitting Laser Diodes, IEEE J. of Quantum Electronics, vol. 31, no. 8 (1995)

[70] L. Raddatz et al., Measurement of Guiding Effects in Vertical-Cavity SurfaceEmitting Lasers, IEEE Photonics Techn. Letters, vol. 8, no. 6 (1996)

[71] M. Lebby et al., Use of VCSEL Arrays for Parallel Optical Interconnects, Proceedings of the SPIE, vol. 2683, pp. 81-91, SPIE, San Jose, CA (1996)

[72] H. Kosaka et al., 2D alignment free VCSEL-array module with push/pill fibre connector, Electr. Lett., vol. 32, no. 21 (1996) 
[73] M. Kajita et al., 1-Gb/s Modulation Characteristics of a Vertical-Cavity Surface-Emitting Laser Array Module, IEEE Photonics Techn. Letters, vol. 9, no. 2 (1997)

[74] M.-L. Andrieux and M. Pearce, Talks given at the LAr optical link meeting at CERN, July 8, 1997

[75] J. Wilson, Talk given at the SCT links meeting at CERN, December 18, 1996

[76] M. Edwards and D.R. Perry, The Radiation Hardness Test Facility, Rutherford Appleton Laboratory, Oxon, England, 1990

[77] M. Edwards, private communication

[78] J.W. Meadows, The ${ }^{9} B e(d, n)$ thick-target neutron spectra for deuteron energies between 2.6 and 7.0 MeV, Nucl. Instr. and Meth. A324 (1993) 239

[79] S. Oglesby, private communication

[80] M. Bosetti et al., DLTS measurement of energetic levels, generated in silicon detectors, Nucl. Instr. and Meth. A361 (1995) 461 